\title{
On unsteady boundary-layer separation in supersonic flow. Part 1. Upstream moving separation point
}

\author{
A. I. RUBAN $N^{1} \dagger, D . A R A K I^{2}, R$. YAPALPAR VI \\ AND J. S. B. GAJJAR \\ ${ }^{1}$ Department of Mathematics, Imperial College London, 180 Queen's Gate, London SW7 2BZ, UK \\ ${ }^{2}$ School of Mathematics, University of Manchester, Oxford Road, Manchester M13 9PL, UK \\ ${ }^{3}$ Department of Mathematics and Statistics, McMaster University, Hamilton, Ontario L8S 4K1, Canada
}

(Received 1 July 2010; revised 25 January 2011; accepted 22 February 2011;

first published online 15 April 2011)

This study is concerned with the boundary-layer separation from a rigid body surface in unsteady two-dimensional laminar supersonic flow. The separation is assumed to be provoked by a shock wave impinging upon the boundary layer at a point that moves with speed $V_{s h}$ along the body surface. The strength of the shock and its speed $V_{s h}$ are allowed to vary with time $t$, but not too fast, namely, we assume that the characteristic time scale $t \ll R e^{-1 / 2} / V_{w}^{2}$. Here $R e$ denotes the Reynolds number, and $V_{w}=-V_{s h}$ is wall velocity referred to the gas velocity $V_{\infty}$ in the free stream. We show that under this assumption the flow in the region of interaction between the shock and boundary layer may be treated as quasi-steady if it is considered in the coordinate frame moving with the shock. We start with the flow regime when $V_{w}=O\left(R e^{-1 / 8}\right)$. In this case, the interaction between the shock and boundary layer is described by classical triple-deck theory. The main modification to the usual triple-deck formulation is that in the moving frame the body surface is no longer stationary; it moves with the speed $V_{w}=-V_{s h}$. The corresponding solutions of the triple-deck equations have been constructed numerically. For this purpose, we use a numerical technique based on finite differencing along the streamwise direction and Chebyshev collocation in the direction normal to the body surface. In the second part of the paper, we assume that $1 \gg V_{w} \gg O\left(R e^{-1 / 8}\right)$, and concentrate our attention on the self-induced separation of the boundary layer. Assuming, as before, that the Reynolds number, $R e$, is large, the method of matched asymptotic expansions is used to construct the corresponding solutions of the Navier-Stokes equations in a vicinity of the separation point.

Key words: boundary layer separation, high-speed flow

\section{Introduction}

L. Prandtl was the first to link the phenomenon of flow separation from a rigid body surface to the behaviour of the boundary layer developing along this surface. In his seminal paper (see Prandtl 1904) read before the third International Mathematics Congress in Heidelberg, he argued that at large values of the Reynolds number, $R e$, almost entire flow field can be treated as inviscid. Still, at any $R e$, no matter 
how large, there always exists a thin layer adjacent to the body surface, termed the boundary layer, where the role of internal viscosity remains of primary importance. The thickness of this layer is estimated to be $O\left(R e^{-1 / 2}\right)$. The behaviour of the boundary layer depends on the pressure distribution along the wall. If the pressure gradient is favourable, i.e. the pressure decreases downstream, then the boundary layer stays attached to the wall. However, with an adverse pressure gradient, when the pressure rises in the direction of the flow, the boundary layer tends to separate from the body surface. The reason for separation may be explained as follows. Since the velocity in the boundary layer drops towards the wall, the kinetic energy of fluid particles inside the boundary layer is less than that at the outer edge of the boundary layer, in fact the closer a fluid particle is to the wall the smaller appears to be its kinetic energy. This means that when the fluid in the outer flow undergoes a deceleration, it can easily produce a pressure rise that is too large for the fluid inside the boundary layer to overcome it. Indeed, even a small increase of pressure may cause the fluid particles near the wall to stop and then turn back to form a reverse flow region characteristic of separated flows.

According to Prandtl, the boundary-layer separation takes place at a position where the skin friction $\tau_{w}$ becomes zero, i.e.

$$
\tau_{w}=\left.\mu \frac{\partial u}{\partial y}\right|_{y=0} .
$$

Here $\mu$ denotes the dynamic viscosity coefficient, $u$ is the velocity component tangential to the wall, and $y$ is the coordinate normal to the wall. In a steady flow past a motionless body, $u=0$ at $y=0$ due to the no-slip condition. Therefore, upstream of the separation, where $\tau_{w}>0$, the fluid velocity $u$ near the wall is positive, which means that the fluid particles in the boundary layer move downstream along the wall, and the flow appears to be attached to the body surface. However, as soon as the skin friction $\tau_{w}$ turns negative, a layer of reversed flow $(u<0)$ emerges near the wall, giving rise to a region of recirculation which, obviously, originates from the separation point defined by (1.1).

Prandtl's description of the separation process, as revealing as it was at the time, still left some important questions unanswered. In particular, it remained unclear why the recirculation flow region did not remain inside the boundary layer whose thickness decreases with the Reynolds number as $O\left(R e^{-1 / 2}\right)$. Instead, experiments show that the eddies erupt from the boundary layer, resulting in the formation of large recirculation regions which influence the entire flow field around the body. This question was answered in the late thirties when it was established that the solution to the boundary-layer equations (in their classical formulation as given by Prandtl) leads to a singularity at the separation point; see Howarth (1938) and Hartree (1939). The form of the singularity was first predicted by Landau \& Lifshitz (1944). Making use of heuristic arguments, they arrived at a conclusion that the skin friction, $\tau_{w}$, decreases when approaching the separation point as

$$
\tau_{w} \sim \sqrt{x_{s}-x}
$$

Here, $x$ is the coordinate measures along the body surface and $x_{s}$ is the position of the separation point. They also found that the velocity component normal to the body surface experiences unbounded growth inversely proportional to $\sqrt{x_{s}-x}$. Later, Goldstein (1948) confirmed this result, and (which is even more important) proved that the singularity at the separation precludes the solution to be continued beyond the point of zero skin friction. 
This clearly shows that the boundary-layer theory in its classical form, as formulated by Prandtl (1904), cannot be used in a vicinity of the separation point. A key element of the separation process, which was not fully appreciated in Prandtl's description, was an interaction between the boundary layer and external inviscid flow, now referred to as the viscous-inviscid interaction. Asymptotic theory of the viscous-inviscid interaction, also known as the triple-deck theory, was formulated simultaneously by Neiland (1969) and Stewartson \& Williams (1969) for the self-induced separation in supersonic flow and by Stewartson (1969) and Messiter (1970) for incompressible fluid flow near the trailing edge of a flat plate. Later, many researchers were involved in the development of the theory, and it became clear that the viscous-inviscid interaction plays a key role in a wide variety of fluid dynamic phenomena. An exposition of applications of the theory to different forms of the boundary-layer separation may be found, for example, in the monograph by Sychev et al. (1998).

The unsteady boundary-layer separation is a significantly more complicated phenomenon, and it may take various forms. Until now, the theoreticians have concentrated on two of these. The first one may be called 'incipient separation', which emerges at a finite time at a particular point on the body surface in an otherwise attached boundary layer. A classical example of such a situation is impulsive motion of a circular cylinder, first considered by Blasius in 1908. Blasius assumed that the cylinder was kept at rest surrounded by a viscous incompressible fluid for all times $t<0$. Then, at time $t=0$, it was suddenly brought to motion with constant velocity $V_{0}$. As long as the boundary layer remains attached to the cylinder surface, the velocity field outside the boundary layer takes the form given by the well-known potential solution of the inviscid incompressible flow theory. In particular, close to the cylinder surface, the tangential velocity appears to be

$$
u=2 V_{0} \sin \theta,
$$

where $\theta$ is the angle measured from the front stagnation point, and $V_{\infty}$ is the freestream velocity. Since (1.3) does not satisfy the no-slip condition, a boundary layer forms immediately on the cylinder surface. At $t=0^{+}$its thickness, $\delta$, is infinitesimal but then it grows as $\delta \sim \sqrt{v t}$, where $v$ is the kinematic viscosity of the fluid. Blasius (1908) analysed the behaviour of the flow through solving the unsteady boundarylayer equations. He sought the series solution in powers of time $t$, and calculated two first terms in the series, the first being

$$
u=\frac{2}{\pi} V_{0} \sin \theta \int_{0}^{y / \sqrt{v t}} \mathrm{e}^{-\zeta^{2} / 4} \mathrm{~d} \zeta+\cdots, \quad \text { as } \quad t \rightarrow 0^{+} .
$$

Further terms were later obtained by Goldstein \& Rosenhead (1936), Wundt (1955), Collins \& Dennis (1973b) and Cowley (1983).

The problem was also studied numerically by finite-differencing the boundarylayer equations, and constructing the solution through marching forward in time. Collins \& Dennis $(1973 a)$, Cebeci $(1979,1982,1986)$ and Wang $(1979,1982)$ applied this approach to the boundary-layer equations written in conventional Euler variables. A new approach was suggested by van Dommelen \& Shen $(1980,1982)$, who wrote the boundary-layer equations in Lagrangian variables before finite-differencing and constructing the numerical solution.

As a result of these works, it has been revealed that after initial growth of the boundary layer described by (1.4), nonlinear effects become important. At $t=0.644$, the skin friction (1.1) becomes zero for the first time, and this occurs at the rear stagnation point, $\theta=180^{\circ}$. As time increases beyond $t=0.644$, the zero skin-friction 
point moves upstream, and a recirculation region forms near the cylinder surface. Unlike in steady flows, the unsteady boundary layer does not develop a singularity at the zero skin-friction point (see, for example, Proudman \& Johnson 1962; Robins \& Howarth 1972; Telionis \& Tsahalis 1974), and therefore, the recirculation region (as it extends upstream from the rear stagnation point) remains confined to the boundary layer. The situation changes drastically at $t \approx 3.0$, when the solution to the boundary-layer equations develops a singularity at position $\theta=111^{\circ}$ that lies within the recirculation region; by this time the point of zero skin friction reaches $\theta=104.5^{\circ}$.

The onset of the singularity signifies the beginning of the separation process. Experimental observations (see, for example, figure 15 in Nakayama 1988) show that as the critical time $t \approx 3.0$ is approached, the boundary layer is lifted off the surface of the cylinder near $\theta=111^{\circ}$ and rolls into a spiral vortex. The latter feeds on the fluid erupting from the boundary layer, grows in size with time and moves away from the cylinder surface.

This form of separation is observed in many other physical situations, most notably, at the leading edge of pitching up aerofoil (see, for example, Degani, Li \& Walker 1996) where the erupting vortex leads to the phenomenon of dynamic stall. Other examples include the boundary-layer separation in front of wall-mounted obstacles (Puhak, Degani, \& Walker 1995) and unsteady separation provoked by a free-stream vortex (Peridier, Smith, \& Walker 1991).

In the second category are flows with 'developed separation'. A typical example is an incompressible fluid flow past a circular cylinder with the Kármán vortex street in its wake. Each individual vortex forms near the cylinder surface through accumulation of vorticity produced by the boundary layer. Once the circulation around the vortex reaches a critical value, it is shed downstream, and another vortex starts to form in its place. During this cycle, the separation point moves up and down the cylinder surface.

Even before calculating the boundary-layer equations for this flow, one can predict that the solution will develop a singularity at the separation point. Indeed, if the solution remained regular, then the separation eddies could stay within the boundary layer, which is not what happens in real flows. In fact, experimental observations show that Kármán vortices are comparable in size with the cylinder. Once the singular behaviour of the boundary layer is excepted, an analogy between unsteady separation over a fixed wall and steady separation on a moving wall can be established (this analogy was mentioned for the first time by Sears 1959 and Moore 1958). Indeed, on approaching the separation point, $\partial u / \partial x$ is expected to become infinitely large, making the convective acceleration of the fluid $u \partial u / \partial x$ much larger than the local acceleration $\partial u / \partial t$, provided that the latter is calculated in the coordinate frame moving with the separation point.

The fact that the flow near the separation point is governed by the steady equations does not yet mean that the theory of steady separation becomes applicable. The fact is that in the frame moving with the separation point, the body surface no longer remains motionless. Let us consider, for example, a part of the vortex shedding cycle when the separation point moves along the cylinder surface upstream and, correspondingly, in the moving frame the cylinder surface moves downstream. Owing to the action of viscous forces, the fluid particles adjacent to the wall will be involved in the downstream motion, which precludes the recirculation region to start from a point on the body surface, as it happens in the case of steady flow separation. Instead, separation now takes place from a point that lies in the middle of the boundary layer. To explain how it happens, let us consider a sequence of cross-sections of the boundary 
layer corresponding to progressively larger values of the longitudinal coordinate $x$. In each cross-section, the fluid velocity $u$ is a function of the normal coordinate $y$. If the boundary layer is exposed to an adverse pressure gradient, then the fluid will experience a deceleration. As a result, the velocity profile across the boundary layer will assume a characteristic form with minimum of $u$ that lies some distance $y_{\min }(x)$ above the wall. If the pressure gradient is strong enough, then the minimal velocity will continue to decrease with $x$, leading to the separation point $\left(x_{s}, y_{\min }\left(x_{s}\right)\right)$, where

$$
u=\frac{\partial u}{\partial y}=0 .
$$

Behind this point, the velocity is expected to become negative on both sides of $y_{\min }$, giving rise to two separation eddies forming in the boundary layer.

The condition (1.5) was suggested by Moore (1958), Rott (1956) and Sears (1956) as a criterion for the separation of steady boundary layer on a moving wall, and since then is referred to as the Moore-Rott-Sears condition. A number of authors (see, for example, Telionis \& Werle 1973) conducted numerical analysis of the boundary layer on the moving wall, and confirmed that the process of the formation of the Moore-Rott-Sears point follows the scenario described above. Experimental study of boundary layer approaching the separation was conducted by Ludwig (1964) and Koromilas \& Telionis (1980). Their measurements also proved to agree, at least qualitatively, with the Moore-Rott-Sears concept.

An important step forward was made by Sychev (1979), who applied the ideas of triple-deck theory to the analysis of steady boundary-layer separation on a downstream moving wall. He confirmed, first of all, that the solution of the classical boundary-layer equations indeed develops a singularity at the Moore-Rott-Sears point (Sychev 1980). Assuming that the pressure gradient remains regular on approach to the separation, he found that the minimum velocity decreases as $u_{\min } \sim \sqrt{x_{s}-x}$. He also proved that the singularity precludes the solution to be continued downstream of the separation, which implies that the boundary-layer theory (in its classical formulation) is insufficient for describing the separation process.

A new theory was developed by Sychev $(1979,1984,1987)$ with help from van Dommelen \& Shen (1983) based on the asymptotic analysis of the Navier-Stokes equations at large values of the Reynolds number. It was shown that, similar to the case of motionless wall (see Sychev 1972), a key element of the separation process is mutual interaction of the boundary layer and external inviscid part of the flow. However, unlike in the conventional triple-deck theory (Sychev 1972), the boundary layer on the downstream moving wall finds itself under the action of extreme adverse pressure gradient even before the interaction region. As a consequence, the flow in the boundary layer experiences a sharp deceleration, leading to the formation of a rather wide region of retarded fluid near the minimal velocity. It completely modifies the process of interaction between the boundary layer and external flow, making it predominantly inviscid. For these and other details of the theory, the reader is referred to chapter 5 of the monograph by Sychev et al. (1998).

Sychev's analysis was concerned with the case of incompressible fluid flow. In this paper, we deal with the supersonic flow separation on a downstream moving wall. We shall show that the supersonic separation proceeds in a way which is significantly different from that in the corresponding subsonic flow. We formulate the problem in $\S 2$ and start, by adopting for our purposes, the classical triple-deck description of the viscous-inviscid interaction. The latter remain valid provided that the wall speed $V_{w}=O\left(R e^{-1 / 8}\right)$. In $\S 3$, the results of the numerical solution of the triple-deck 


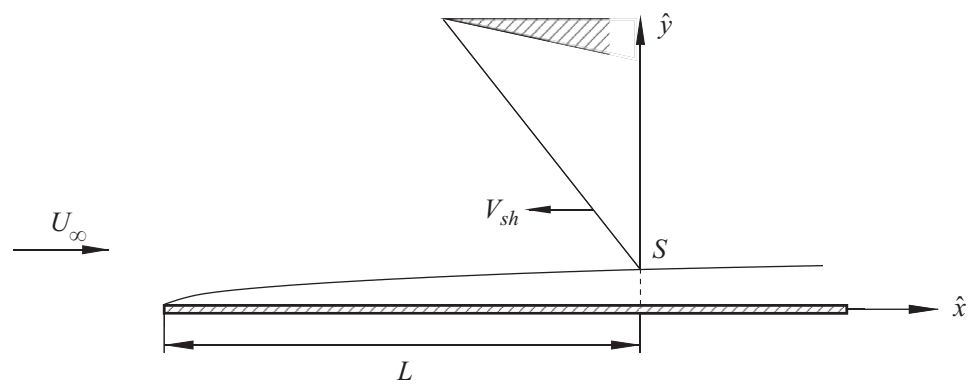

Figure 1. The flow layout.

equations are presented. Then, in $\S 4$ we turn to asymptotic analysis of the flow which is conducted on assumption that $V_{w} \gg R e^{-1 / 8}$. In $\S 4.1$, the main region of interaction between the boundary layer and the inviscid external flow is considered. The interaction acts to smooth out the pressure jump in the impinging shock. In the flow considered, the longitudinal extent of the interaction region proves to be relatively small, which makes the flow inviscid not only outside but also inside the boundary layer. The solution of the interaction problem allows determination of the pressure distribution along the body surface. However, this solution does not satisfy the no-slip condition, which is why in $\S 4.2$ we introduce a thinner layer closer to the wall. The flow in this layer is viscous and is described by Prandtl's boundarylayer equations. It appears that the solution to these equations develops a singularity characteristic of a separation process. Unusually, the singularity forms at the outer edge of the viscous layer. In $\S 4.3$, we study displacement effect of the viscous layer. We find that the displacement thickness of the layer amplifies as $O(\ln s / s)$, where $s$ is the distance from the singular point. Using this result, we show in $\S 4.4$ that there exists a vicinity of the singular point where description of the interaction process has to be modified to account for a contribution of the viscous layer. Interestingly enough, in this new region the flow remains inviscid, and therefore, the governing equations only allow to describe the flow deceleration towards the separation, but not a formation of recirculation eddies. The latter task is performed in $\S 4.5$, where a small viscous region surrounding the separation point is analysed. The solution for this region (and, in fact, all other regions) can be found in an analytic form.

\section{Problem formulation}

Let a flat plate be placed in supersonic flow of a perfect gas parallel to the free-stream velocity vector. Furthermore, let a shock wave be generated by a wedge situated above the plate as shown in figure 1 . We shall assume that the wedge, and hence the shock, are moving upstream with velocity $V_{s h}$.

We denote by $L$ the distance between the leading edge of the plate and the current position of point $S$, where the shock impinges upon the boundary layer; the velocity, density, viscosity and pressure in the unperturbed flow upstream of the plate are denoted by $V_{\infty}, \rho_{\infty}, \mu_{\infty}$ and $p_{\infty}$, respectively. To study the flow, we shall use Cartesian coordinates with $\hat{x}$ measured from the point $S$ along the plate surface, and $\hat{y}$ in the perpendicular direction. The velocity components in these coordinates are denoted by $\hat{u}$ and $\hat{v}$. We shall further denote the gas density by $\hat{\rho}$, pressure $\hat{p}$, enthalpy $\hat{h}$ and 
viscosity $\hat{\mu}$. The 'hat' is used here to indicate that the corresponding variables are dimensional. The non-dimensional variables are introduced as

$$
\left.\begin{array}{l}
\hat{u}=V_{\infty} u, \quad \hat{v}=V_{\infty} v, \quad \hat{\rho}=\rho_{\infty} \rho, \\
\hat{p}=p_{\infty}+\rho_{\infty} V_{\infty}^{2} p, \quad \hat{h}=V_{\infty}^{2} h, \quad \hat{\mu}=\mu_{\infty} \mu,
\end{array}\right\}
$$

In the analysis that follows we assume that the Reynolds number

$$
R e=\frac{\rho_{\infty} V_{\infty} L}{\mu_{\infty}} \rightarrow \infty .
$$

We shall also assume that the free-stream Mach number

$$
M_{\infty}=\frac{V_{\infty}}{\sqrt{\gamma \frac{p_{\infty}}{\rho_{\infty}}}}
$$

has a finite value larger than one; in the above formula, $\gamma$ denotes the specific heat ratio of the gas considered.

To start with, we shall consider the case when the shock speed

$$
V_{\text {sh }}=O\left(R e^{-1 / 8}\right) .
$$

In this case, the interaction of the shock wave with the boundary layer may be described using the classical triple-deck theory. The formulation of the triple-deck equations has been discussed in numerous publications including, of course, the original papers of Neiland (1969) and Stewartson \& Williams (1969). We, therefore, will give here only a brief account of the theory (for more details, see Battam et al. 2004).

As the shock impinges upon the boundary layer, causing the pressure to rise in the downstream direction, the gas inside the boundary layer undergoes a deceleration. This leads to a thickening of flow filaments in the subsonic part of the boundary layer near the plate surface, while those in the supersonic part near the outer edge of the boundary layer are made thinner. Still, since the slower-moving gas is more sensitive to pressure variation, the integral effect is the displacement of the streamlines from the plate surface. In response, the supersonic flow outside the boundary layer produces a family of compression waves. As a result, the jump in the impinging shock is converted into a smooth pressure rise, which adjusts itself to the flow deceleration in the process termed the viscous-inviscid interaction.

On the basis of the asymptotic analysis of the Navier-Stokes equations with $R e \rightarrow \infty$, Neiland (1969) and Stewartson \& Williams (1969) demonstrated that the region of interaction is $O\left(R^{-3 / 8}\right)$ long and has a three-tiered structure as shown in figure 2. It is composed of the viscous sublayer (region 1 in figure 2), the main part of the boundary layer (region 2) and an inviscid potential flow region 3 situated outside the boundary layer. The characteristic thickness of the viscous sublayer is estimated as being an $O\left(R e^{-5 / 8}\right)$ quantity, so that it occupies an $O\left(R e^{-1 / 8}\right)$ portion of the boundary layer and is comprised of the stream filaments immediately adjacent to the wall. The flow velocity in this region is $O\left(R e^{-1 / 8}\right)$ relative to the free-stream velocity, 


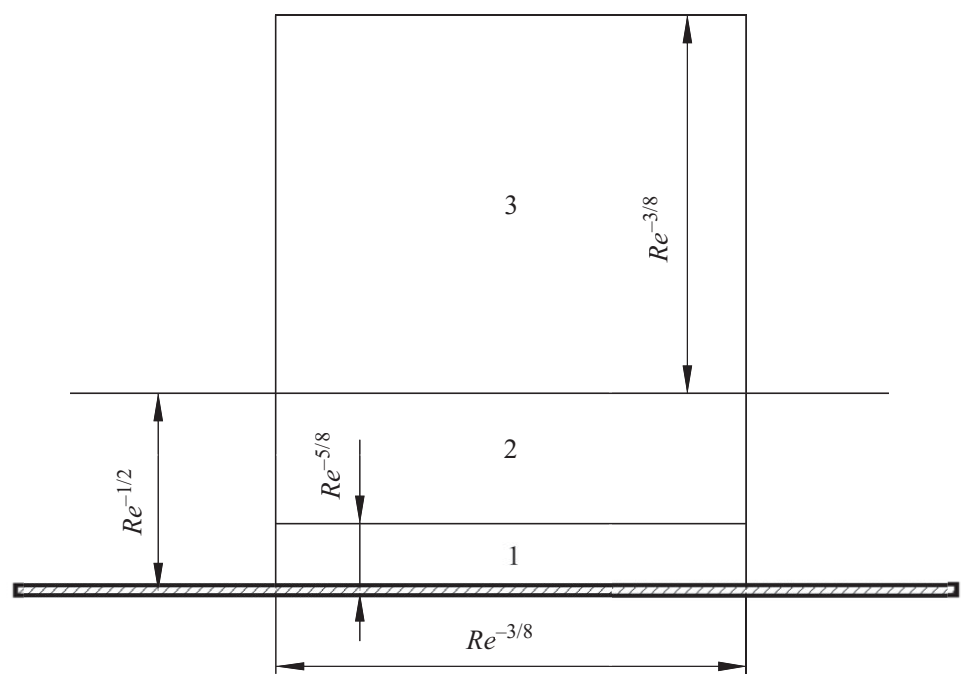

FIGURE 2. Three-tiered structure of the interaction region.

and due to the slow motion of gas here, the flow exhibits high sensitivity to pressure variations. Even a small variation of pressure along the wall may cause significant deceleration/acceleration of fluid particles there. As a result, the flow filaments change their thickness, leading to the deformation of streamlines. This process is termed the displacement effect of the boundary layer.

The main part of the boundary layer, the middle tier of the interactive structure, represents a continuation of the conventional boundary layer developing along the plate. Its thickness is estimated as $O\left(R e^{-1 / 2}\right)$ and the velocity is an order-one quantity. The flow in this tier is significantly less sensitive to the pressure variations. It does not produce any noticeable contribution to the displacement effect of the boundary layer, which means that all the streamlines in the middle tier are parallel to each other and carry the deformation produced by the displacement effect of the viscous sublayer.

Finally, the upper tier is situated in the potential flow region outside the boundary layer. It serves to 'convert' the perturbations in the form of the streamlines into perturbations of pressure. These are then transmitted through the main part of the boundary layer back to the sublayer. As the velocity in the viscous sublayer $u=O\left(R e^{-1 / 8}\right)$, the flow separation may be expected to take place when the pressure rise in the impinging shock reaches a critical value of order

$$
\Delta p_{\text {shock }} \sim u^{2} \sim R e^{-1 / 4} .
$$

Under the assumptions (2.5) and (2.6), the asymptotic solution of the Navier-Stokes equations in the viscous sublayer (region 1) may be sought in the form

$$
\left.\begin{array}{ll}
x=1+\operatorname{Re}^{-3 / 8} \frac{\mu_{w}^{-1 / 4} \rho_{w}^{-1 / 2}}{\lambda^{5 / 4} \beta^{3 / 4}} X, & y=\operatorname{Re}^{-5 / 8} \frac{\mu_{w}^{1 / 4} \rho_{w}^{-1 / 2}}{\lambda^{3 / 4} \beta^{1 / 4}} Y, \\
u=\operatorname{Re}^{-1 / 8} \frac{\mu_{w}^{1 / 4} \rho_{w}^{-1 / 2}}{\lambda^{-1 / 4} \beta^{1 / 4}} U+\cdots, & v=\operatorname{Re}^{-3 / 8} \frac{\mu_{w}^{3 / 4} \rho_{w}^{-1 / 2}}{\lambda^{-3 / 4} \beta^{-1 / 4}} V+\cdots, \\
p=\operatorname{Re}^{-1 / 4} \frac{\mu_{w}^{1 / 2} \rho_{w}^{-1 / 2}}{\lambda^{-1 / 2} \beta^{1 / 2}} P+\cdots, & \rho=\rho_{w}+\cdots, \quad \mu=\mu_{w}+\cdots .
\end{array}\right\}
$$


Here in addition to conventional scaling with respect to the small parameter $R e^{-1 / 8}$, we use affine transformations with $\rho_{w}$ and $\mu_{w}$ being the dimensionless density and dynamic viscosity of the gas on the plate surface immediately before the interaction region; the dependence of the solution on the free-stream Mach number, $M_{\infty}$, is expressed through the parameter $\beta=\sqrt{M_{\infty}^{2}-1}$. The affine transformations allow one to reduce the equations for the interaction region to the following canonical form independent of the values of $\rho_{w}, \mu_{w}$ and $\beta$.

The flow in region 1 is described by the boundary-layer equations

$$
\begin{aligned}
U \frac{\partial U}{\partial X}+V \frac{\partial U}{\partial Y} & =-\frac{\mathrm{d} P}{\mathrm{~d} X}+\frac{\partial^{2} U}{\partial Y^{2}}, \\
\frac{\partial U}{\partial X}+\frac{\partial V}{\partial Y} & =0 .
\end{aligned}
$$

Here, the pressure $P$ does not change with $Y$, being a function of $X$ only. Equations (2.8a) and (2.8b) have to be solved with the no-slip conditions on the plate surface

$$
U=U_{w}, \quad V=0 \quad \text { at } \quad Y=0 .
$$

Note that in the coordinate frame moving with the shock (see figure 1), the plate surface moves in the opposite direction. We denote its speed, scaled with $R^{-1 / 8}$, as $U_{w}$.

Equations (2.8a) and (2.8b) also require the initial condition

$$
U=Y+U_{w} \text { at } X=-\infty,
$$

which follows from matching with the solution in the unperturbed boundary layer upstream of the interaction region.

The matching with the solution in the main part of the boundary layer (region 2 in figure 2) yields

$$
U \rightarrow Y+A(X)+\cdots \quad \text { as } \quad Y \rightarrow \infty,
$$

where $A(X)$ denotes the so-called displacement function. It is used in the interaction law, which is deduced from the flow analysis in region 3 (see figure 2),

$$
P=P_{s} \mathscr{H}(X)-\frac{\mathrm{d} A}{\mathrm{~d} X} .
$$

Here the first term represents the impinging shock, $P_{s}$ denotes the pressure jump in the shock scaled with $R e^{-1 / 4}$, and $\mathscr{H}$ denotes the Heaviside function

$$
\mathscr{H}(X)= \begin{cases}0, & \text { if } X<0 \\ 1, & \text { if } X \geqslant 0 .\end{cases}
$$

The second term in (2.8f) represents the displacement effect of the boundary layer.

Finally, we have to take into account the fact that viscous-inviscid interaction allows upstream influence through the boundary layer even in well-attached flows (see Lighthill 1953). This means that an additional boundary condition specifying the state of the flow downstream of the interaction region is required. We shall assume that

$$
P=P_{s} \quad \text { at } \quad X=\infty .
$$

This condition closes the formulation of the interaction problem (2.8). 


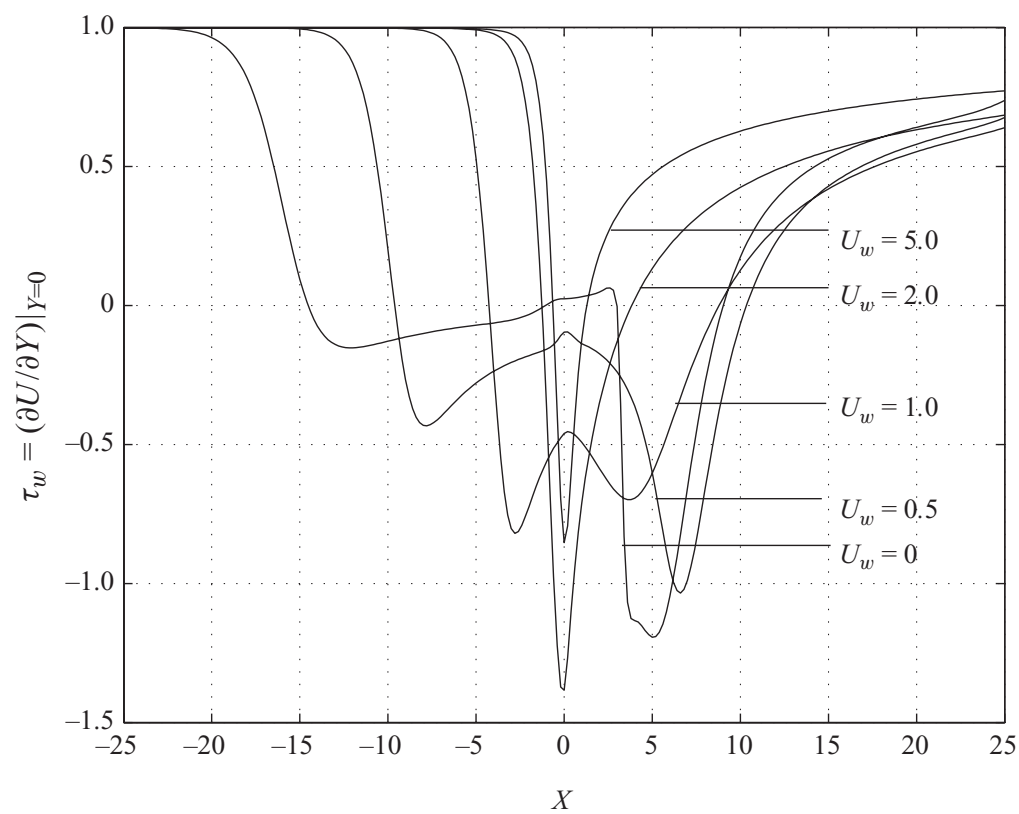

FIGURE 3. Skin friction $\tau_{w}$ for the shock strength $P_{s}=5$ and various values of the wall velocity $U_{w}$.

\section{Computational results}

Here the results of the numerical solution of the interaction problem (2.8) are presented. The calculations were performed using method B as described by Korolev, Gajjar \& Ruban (2002). In this method, (2.8) are first written in terms of the streamfunction $\Psi$ using $U=\partial \Psi / \partial Y$ and $V=-\partial \Psi / \partial X$. Then, the derivatives with respect to $X$ are represented using windward finite differences. In the $Y$-direction, the domain $0<Y<Y_{\max }$ was mapped linearly into $-1<z<1$, and at each $X$-station, Chebychev collocation was used to satisfy the equations and boundary conditions. The resulting set of algebraic equations was solved with the help of Newton iterations.

The results shown below were obtained on the computational domain which extended in the streamwise direction from $X_{\min }=-60$ to $X_{\max }=-60$; a uniform grid was used with $\Delta X=0.01$. In the $Y$-direction, 64 collocation points were used, and the outer boundary was placed at $Y_{\max }=50$. As usual, a smoothing was applied to the impinging shock, namely, for computational purposes the interaction law $(2.8 f)$ was written as

$$
P=\frac{P_{s}}{2}\left(1+\frac{X}{\sqrt{X^{2}+r^{2}}}\right)-\frac{\mathrm{d} A}{\mathrm{~d} X} .
$$

In this study, parameter $r$ was chosen to be $r=0.5$.

The influence of the wall motion on the flow in the interaction region is demonstrated in figures 3 and 4 . For the motionless wall $\left(U_{w}=0.0\right)$, most revealing is the skin friction plot (see figure 3). One can see that there are four points where the $\tau_{w}(X)$ curve intersects the abscissa. The first and the last of these give the separation and reattachment positions of the primary separation bubble. The other two correspond to the second smaller separation bubble embedded inside the primary 


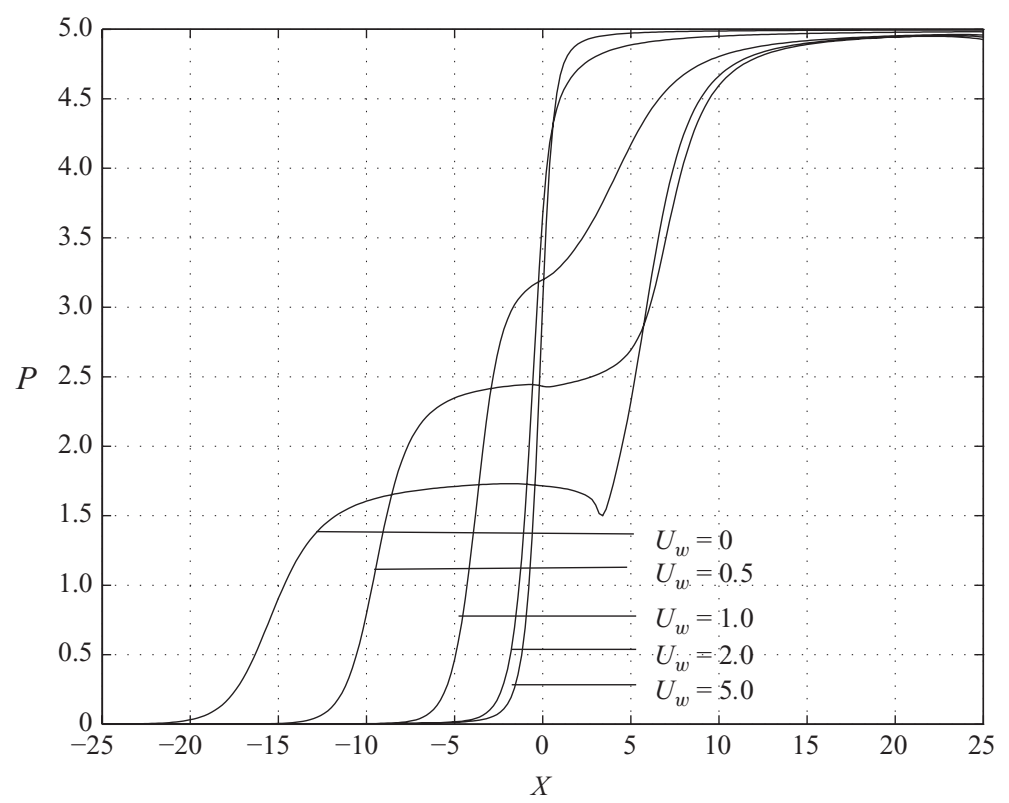

Figure 4. Pressure $P$ for the shock strength $P_{s}=5$ and various values of the wall velocity $U_{w}$.

recirculation region. As the flow in the recirculation region is rather slow, the pressure plot (see figure 4) develops a characteristic 'plateau'.

When the wall starts moving, the skin friction no longer shows where the recirculation region is; it rather shows if the fluid layer adjacent to the wall moves faster $\left(\tau_{w}>0\right)$ or slower $\left(\tau_{w}<0\right)$ than the wall itself. Still, there are various indicators in the skin friction and pressure behaviour that can be used to judge whether the flow is separated or not. When the flow is separated, the skin friction has two minima; the first being situated immediately behind the separation point and the second in front of the reattachment. Also, the pressure retains the plateau, which can be seen for the wall speed of up to $U_{w}=1.0$. When the wall speed is increased to $U_{w}=2.0$, the skin friction shows only one minimum (see figure 3), and the plateau disappears from the pressure plot (see figure 4).

Of course, in order to determine if the boundary layer is fully attached or a separation region is embedded in the flow, a more detailed analysis of the flow field is required. The transition between attached and separated forms of the flow takes place when the longitudinal velocity $U$ is positive everywhere except for one point with $U=0$. At this point, the condition (1.5) obviously holds. In particular, for the shock strength $P_{s}=5$, the wall speed has to be increased to the value of $U_{w}=1.18$ in order to shrink the recirculation region to a point. For $P_{s}=7$, the corresponding value of $U_{w}$ appears to be 1.97; if $U_{w}<1.97$, then the boundary-layer flow has an impeded separation bubble. Its structure is shown in figure 5 for $P_{s}=7$ and $U_{w}=0.2$. We see that the separation manifests itself through a saddle point in the streamline pattern, where the Moore-Rott-Sears condition (1.5) holds. Behind this point, two recirculation regions form, one sitting on top of the other. The fluid in these regions rotates in the opposite directions; in the upper region the rotation is clockwise, in the lower anticlockwise. The separation is terminated by the second saddle point. Note that above this point an additional vortex starts to form. 


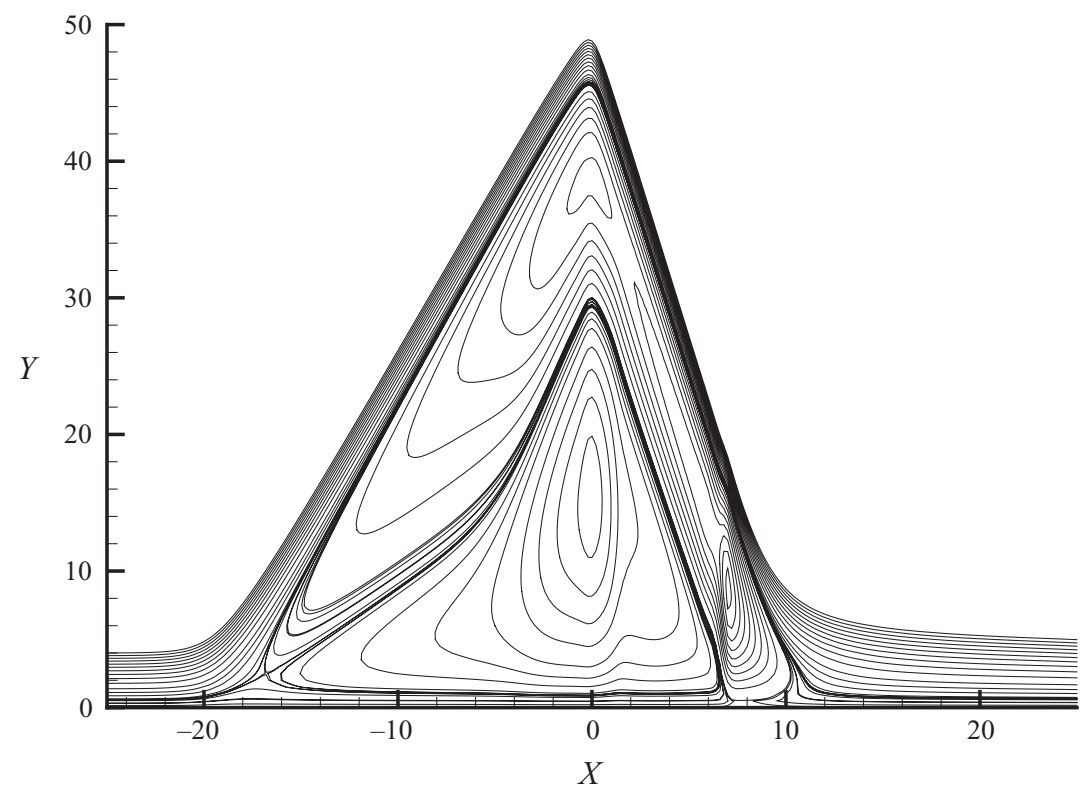

FIGURE 5. Streamline pattern for $P_{s}=7$ and $U_{w}=0.5$.

\section{Asymptotic theory for large values of the wall speed $U_{w}$}

If the wall speed $U_{w}$ in the no-slip condition $(2.8 c)$ is large, then the investigation of the separation process may be conducted theoretically. This may be done based on the asymptotic analysis of the Navier-Stokes equations under the assumption that the wall speed is small as compared to the free-stream velocity $V_{\infty}$ but large as compared to $V_{\infty} R e^{-1 / 8}$. Alternatively, one can start with the triple-deck problem (2.8) and assume that $U_{w} \rightarrow \infty$. In this presentation, we adopt the second approach.

For the theoretical study, it is convenient to write the interaction problem (2.8) in von Mises variables,

$$
\begin{gathered}
U \frac{\partial U}{\partial X}+\frac{\mathrm{d} P}{\mathrm{~d} X}=U \frac{\partial}{\partial \Psi}\left(U \frac{\partial U}{\partial \Psi}\right), \\
\frac{\partial}{\partial \Psi}\left(\frac{V}{U}\right)=\frac{\partial}{\partial X}\left(\frac{1}{U}\right), \\
P=P_{s} \mathscr{H}(X)+\lim _{\Psi \rightarrow \infty} \frac{V}{U}, \\
U=U_{w}, \quad V=0, \quad \text { at } \quad \Psi=0, \\
U=\sqrt{2 \Psi+U_{w}^{2}}, \quad \text { at } \quad X=-\infty, \\
U \rightarrow \sqrt{2 \Psi}+\cdots, \quad \text { as } \quad \Psi \rightarrow \infty, \\
P=P_{s}, \quad \text { at } \quad X=\infty .
\end{gathered}
$$

Here, $\Psi$ is the streamfunction defined by the equations

$$
\frac{\partial \Psi}{\partial X}=-V, \quad \frac{\partial \Psi}{\partial Y}=U .
$$


We shall see that as $U_{w} \rightarrow \infty$, the lower deck (region 1) of the triple-deck structure (see figure 2) splits into two regions, the upper layer, region $1 a$, and the near-wall layer, region $1 b$.

\subsection{Region $1 a$}

The form of the asymptotic solution of the interaction problem (4.1) in region $1 a$ may be determined using the principle of least degeneration. We start with the initial condition (4.1e). In order to avoid degeneration in (4.1e), we have to assume that

$$
\Psi \sim U_{w}^{2} \text { and } U \sim U_{w}
$$

Since the separation is a nonlinear process, we have to ensure that the two terms on the left-hand side of the momentum equation (4.1) remain in balance with each other. This is possible only if

$$
P \sim U^{2} \sim U_{w}^{2}
$$

An estimate for the lateral velocity component $V$ is obtained, as usual, from the continuity equation $(4.1 b)$. The two sides of this equation remain in balance with each other provided that

$$
V \sim \frac{U_{w}^{2}}{\Delta X}
$$

Here, $\Delta X$ is the longitudinal scale of the interaction region. It may be found using the interaction law $(4.1 c)$. Balancing the pressure on the left-hand side of $(4.1 c)$ with the second term on the right-hand side, it is easily found that

$$
\Delta X \sim \frac{1}{U_{w}} .
$$

Substituting (4.6) back into (4.5), we find

$$
V \sim U_{w}^{3}
$$

This suggests that the asymptotic solution of (4.1) in region $1 a$ may be sought in the form

$$
\left.\begin{array}{c}
X=\frac{1}{U_{w}} X_{*}, \quad \Psi=U_{w}^{2} \Psi_{*}, \\
U=U_{w} U_{*}+\cdots, \quad V=U_{w}^{3} V_{*}+\cdots, \quad P=U_{w}^{2} P_{*}+\cdots .
\end{array}\right\}
$$

Substitution of (4.8) into (4.1) results in

$$
\begin{gathered}
U_{*} \frac{\partial U_{*}}{\partial X_{*}}+\frac{\mathrm{d} P_{*}}{\mathrm{~d} X_{*}}=0, \\
\frac{\partial}{\partial \Psi_{*}}\left(\frac{V_{*}}{U_{*}}\right)=\frac{\partial}{\partial X_{*}}\left(\frac{1}{U_{*}}\right), \\
P_{*}=\bar{P}_{s} \mathscr{H}\left(X_{*}\right)+\lim _{\Psi_{*} \rightarrow \infty} \frac{V_{*}}{U_{*}}, \\
V_{*}=0, \quad \text { at } \quad \Psi_{*}=0, \\
U_{*}=\sqrt{2 \Psi_{*}+1}, \quad \text { at } \quad X_{*}=-\infty, \\
U_{*} \rightarrow \sqrt{2 \Psi_{*}}+\cdots, \quad \text { as } \quad \Psi_{*} \rightarrow \infty, \\
P_{*}=\bar{P}_{s}, \quad \text { at } \quad X_{*}=\infty .
\end{gathered}
$$


Here it has been assumed that the shock strength

$$
P_{s}=U_{w}^{2} \bar{P}_{s},
$$

with $\bar{P}_{s}$ being an order-one parameter. Note that the viscous term disappears from the momentum equation (4.9a), signifying that the flow in region $1 a$ should be treated as inviscid. Therefore, we can only impose the impermeability condition $(4.9 d)$ on the body surface, leaving the task of satisfying the no-slip condition to the flow analysis in region $1 b$.

Integration of $(4.9 a)$ leads to the Bernoulli equation

$$
\frac{1}{2} U_{*}^{2}+P_{*}=H_{*}\left(\Psi_{*}\right),
$$

where the function $H_{*}\left(\Psi_{*}\right)$ may be found from the initial condition $(4.9 e)$ to be

$$
H_{*}\left(\Psi_{*}\right)=\Psi_{*}+\frac{1}{2} .
$$

Substituting (4.12) back into (4.11), and solving the resulting equation for $U_{*}$, we find

$$
U_{*}=\sqrt{2 \Psi_{*}+1-2 P_{*}} .
$$

Now we can use (4.13) on the right-hand side of $(4.9 b)$ which, being integrated with respect to $\Psi_{*}$, yields

$$
\frac{V_{*}}{U_{*}}=\left(\frac{1}{\sqrt{1-2 P_{*}}}-\frac{1}{\sqrt{2 \Psi_{*}+1-2 P_{*}}}\right) \frac{\mathrm{d} P_{*}}{\mathrm{~d} X_{*}} .
$$

It remains to set $\Psi_{*}=\infty$ in (4.14), and substitute the result into the interaction law $(4.9 c)$. This leads to the following equation for the pressure:

$$
P_{*}=\bar{P}_{s} \mathscr{H}\left(X_{*}\right)+\frac{1}{\sqrt{1-2 P_{*}}} \frac{\mathrm{d} P_{*}}{\mathrm{~d} X_{*}} .
$$

We shall start with the region behind the shock $\left(X_{*}>0\right)$, where (4.15) assumes the form

$$
P_{*}-\bar{P}_{s}=\frac{1}{\sqrt{1-2 P_{*}}} \frac{\mathrm{d} P_{*}}{\mathrm{~d} X_{*}} .
$$

It is easily seen that the solution to (4.16) satisfying the boundary condition $(4.9 \mathrm{~g})$ is simply

$$
P_{*}=\bar{P}_{s} \quad \text { if } \quad X_{*} \geqslant 0 .
$$

Indeed, assuming $P_{*}>\bar{P}_{s}$ at some point $X_{*}>0$ makes the derivative $\mathrm{d} P_{*} / \mathrm{d} X_{*}$ positive according to (4.16). Therefore, instead of decreasing towards $\bar{P}_{s}$, the pressure $P_{*}$ could only increase with $X_{*}$. Similarly, if we start with $P_{*}<\bar{P}_{s}$, then the derivative $\mathrm{d} P_{*} / \mathrm{d} X_{*}$ appears to be negative, and the solution will deviate further from the asymptote $(4.9 g)$.

In front of the shock $\left(X_{*}<0\right),(4.15)$ is written as

$$
P_{*}=\frac{1}{\sqrt{1-2 P_{*}}} \frac{\mathrm{d} P_{*}}{\mathrm{~d} X_{*}} .
$$

It can be easily solved by introducing a new unknown function $\Phi=\sqrt{1-2 P_{*}}$, which turns (4.18) into

$$
\frac{\mathrm{d} \Phi}{\mathrm{d} X_{*}}=\frac{\Phi^{2}-1}{2} .
$$


The general solution of (4.19) is

$$
\Phi=\frac{1-C \mathrm{e}^{X_{*}}}{1+C \mathrm{e}^{X_{*}}}
$$

with the corresponding solution for the pressure, $P_{*}$, being

$$
P_{*}=\frac{1}{2}\left[1-\left(\frac{1-C \mathrm{e}^{X_{*}}}{1+C \mathrm{e}^{X_{*}}}\right)^{2}\right] \quad \text { if } \quad X_{*}<0 .
$$

Here $C$ denotes the integration constant.

The value of constant $C$ may be determined through the following line of arguments. Equation (4.18) shows that if the pressure $P_{*}$ is positive, then it has to grow with $X_{*}$, but if $P_{*}$ is negative then it has to decay with $X_{*}$. Correspondingly, all solutions of (4.18) may be divided into two families, the 'compression family' with growing $P$ * and 'expansion family' with decaying $P_{*}$.

Setting $X_{*} \rightarrow-\infty$ in (4.21) results in

$$
P_{*}=2 C \mathrm{e}^{X_{*}}+\cdots \quad \text { as } \quad X_{*} \rightarrow-\infty,
$$

which shows that the compression solutions (in which we are interested) correspond to positive values of $C$. It can be further noted that for any $C>0$, there exists a constant, $\chi$, such that

$$
C=\mathrm{e}^{-\chi}
$$

Substitution of (4.23) into (4.21) yields

$$
P_{*}=\frac{1}{2}\left[1-\left(\frac{1-\mathrm{e}^{X_{*}-\chi}}{1+\mathrm{e}^{X_{*}-\chi}}\right)^{2}\right],
$$

and we can conclude that each member of the 'compression family' may be obtained from another one by a simple shift in $X_{*}$.

The value of the shift parameter, $\chi$, is determined from the condition that the pressure $P^{*}$ is continuous at $X^{*}=0$, namely, that at $X_{*}=0$ the value of $P^{*}$, as given by (4.24), coincides with that given by (4.17),

$$
\frac{1}{2}\left[1-\left(\frac{1-\mathrm{e}^{-\chi}}{1+\mathrm{e}^{-\chi}}\right)^{2}\right]=\bar{P}_{s}
$$

There are two solutions to (4.25),

$$
\chi_{1}=\ln \frac{1+\sqrt{1-2 \bar{P}_{s}}}{1-\sqrt{1-2 \bar{P}_{s}}}, \quad \chi_{2}=\ln \frac{1-\sqrt{1-2 \bar{P}_{s}}}{1+\sqrt{1-2 \bar{P}_{s}}} .
$$

The first of these, $\chi_{1}$, is positive, and the second, $\chi_{2}$, is negative.

If we substitute (4.23) into (4.20), we will have

$$
\Phi=\frac{1-\mathrm{e}^{X_{*}-\chi}}{1+\mathrm{e}^{X_{*}-\chi}} .
$$

By virtue of the definition of function $\Phi$, it has to be positive for all $X_{*} \in(-\infty, 0]$, which is possible only if $\chi>0$. Consequently, the negative root, $\chi_{2}$, of (4.25) has to be discarded in favour of the positive root, $\chi_{1}$.

Let us now return to (4.13). It shows that as soon as $P_{*}$ reaches the value of $1 / 2$, the velocity at the bottom of region $1 a$, where $\Psi_{*}=0$, becomes zero. We shall see that the solution for region $1 b$ develops a singularity at this point, characteristic of the 
separation process. We, therefore, shall assume that $\bar{P}_{s}=1 / 2$, in which case $\chi_{1}=0$, and (4.24) turns into

$$
P_{*}= \begin{cases}\frac{1}{2}\left[1-\left(\frac{1-\mathrm{e}^{X_{*}}}{1+\mathrm{e}^{X_{*}}}\right)^{2}\right], & \text { if } X_{*}<0, \\ \frac{1}{2}, & \text { if } X_{*} \geqslant 0 .\end{cases}
$$

It should be noted that (4.28) is also applicable for the case of 'self-induced separation' caused, say, by a shock wave stronger than $\bar{P}_{s}=1 / 2$, which impinges upon the boundary layer some distance downstream of the interaction region.

To conclude the analysis of region $1 a$, we substitute (4.28) into (4.13) and choose $\Psi_{*}=0$. As a result, we find that the velocity at the outer edge of region $1 b$ is given by

$$
U_{e}\left(X_{*}\right)= \begin{cases}\frac{1-\mathrm{e}^{X_{*}}}{1+\mathrm{e}^{X_{*}}}, & \text { if } X_{*}<0, \\ 0, & \text { if } X_{*} \geqslant 0 .\end{cases}
$$

\subsection{Region $1 b$}

In region $1 b$, the asymptotic solution of the interaction problem (4.1) is sought in the form

$$
U=U_{w} \widetilde{U}\left(X_{*}, \Psi\right)+\cdots, \quad V=U_{w} \widetilde{V}\left(X_{*}, \Psi\right)+\cdots, \quad \text { as } \quad U_{w} \rightarrow \infty .
$$

Here, it is taken into account that region $1 b$ has the same longitudinal extent as region $1 a$. The scaling for $U$ is dictated by the necessity to perform the matching with the solution in region $1 a$. We also need to satisfy the no-slip condition $(4.1 d)$ on the body surface. The latter is possible only if the convective term in the momentum equation $(4.1 a)$ remains in balance with the viscous term,

$$
U \frac{\partial U}{\partial X} \sim U \frac{\partial}{\partial \Psi}\left(U \frac{\partial U}{\partial \Psi}\right) .
$$

Since $X \sim U_{w}^{-1}$ and $U \sim U_{w}$, it follows from (4.31) that $\Psi=O(1)$.

As usual, an estimate for the lateral velocity component, $V$, is found by balancing the two terms in the continuity equation $(4.1 b)$. We find that $V \sim 1 / X \sim U_{w}$. Finally, we note that the pressure, $P$, in region $1 b$ remains the same as in region $1 a$. This means that the leading-order term of the asymptotic expansion for $P$ has the form

$$
P=U_{w}^{2} P_{*}\left(X_{*}\right)+\cdots, \quad \text { as } \quad U_{w} \rightarrow \infty,
$$

with $P_{*}$ given by (4.28).

Substitution of (4.30) and (4.32) into (4.1) leads to

$$
\begin{aligned}
& \widetilde{U} \frac{\partial \widetilde{U}}{\partial X_{*}}-U_{e} \frac{\mathrm{d} U_{e}}{\mathrm{~d} X_{*}}=\widetilde{U} \frac{\partial}{\partial \Psi}\left(\widetilde{U} \frac{\partial \widetilde{U}}{\partial \Psi}\right), \\
& \frac{\partial}{\partial \Psi}\left(\frac{\widetilde{V}}{\widetilde{U}}\right)=\frac{\partial}{\partial X_{*}}\left(\frac{1}{\widetilde{U}}\right), \\
& \widetilde{U}=1, \quad \widetilde{V}=0, \quad \text { at } \quad \Psi=0, \\
& \widetilde{U}=1, \quad \text { at } \quad X_{*}=-\infty, \\
& \widetilde{U}=U_{e}\left(X_{*}\right), \quad \text { at } \quad \Psi=\infty .
\end{aligned}
$$


Here, we have used the fact that the pressure (4.28) and the velocity (4.29) at the outer edge of region $1 b$ are related to each other through the Bernoulli equation (4.11).

Our task will be to determine the behaviour of the solution of (4.33) near the outer edge of region $1 b$ on approaching $X_{*}=0$, where $U_{e}$ becomes zero. To perform this task, we have to start with the asymptotic analysis of (4.33) as $X_{*} \rightarrow-\infty$. It follows from (4.29) that

$$
U_{e}=1-2 \mathrm{e}^{X_{*}}+\cdots \quad \text { as } \quad X_{*} \rightarrow-\infty .
$$

Taking this into account, we seek the longitudinal velocity, $\widetilde{U}$, inside region $1 b$ in the form

$$
\widetilde{U}=1+f(\Psi) \mathrm{e}^{X_{*}}+\cdots, \quad \text { as } \quad X_{*} \rightarrow-\infty, \quad \Psi=O(1) .
$$

Substitution of (4.35) and (4.34) into (4.33a), (4.33c) and (4.33e) results in the following boundary-value problem for the function $f(\Psi)$ :

$$
f^{\prime \prime}-f=2, \quad f(0)=0, \quad f(\infty)=-2 .
$$

Its solution is easily found to be

$$
f(\Psi)=-2+2 \mathrm{e}^{-\Psi} .
$$

Substitution of (4.37) back into (4.35) yields

$$
\widetilde{U}=1-2 \mathrm{e}^{X_{*}}+2 \mathrm{e}^{X_{*}-\Psi}+\cdots .
$$

Let us now consider the limit

$$
\Psi \rightarrow \infty, \quad X^{*}=O(1) .
$$

At this stage, it is convenient to introduce a modified Bernoulli function

$$
\widetilde{H}=\frac{1}{2} \widetilde{U}^{2}-\frac{1}{2} U_{e}^{2},
$$

which allows one to write $(4.33 a)$ in the form

$$
\frac{\partial \widetilde{H}}{\partial X_{*}}=\widetilde{U} \frac{\partial^{2} \widetilde{H}}{\partial \Psi^{2}} .
$$

Substitution of (4.38) and (4.34) into (4.40) shows that

$$
\widetilde{H}=2 \mathrm{e}^{X_{*}-\Psi}+\cdots, \quad \text { as } \quad X_{*} \rightarrow-\infty, \quad \Psi=O(1) .
$$

This suggests that for finite values of $X_{*}$, the solution of (4.41) should be sought near the outer edge of region $1 b$ in the form

$$
\widetilde{H}\left(X_{*}, \Psi\right)=h\left(X_{*}\right) \mathrm{e}^{-\Psi}+\cdots, \quad \text { as } \quad \Psi \rightarrow \infty,
$$

where the function $h\left(X_{*}\right)$ is such that

$$
h\left(X_{*}\right)=2 \mathrm{e}^{X_{*}}+\cdots, \quad \text { as } \quad X_{*} \rightarrow-\infty .
$$

Substituting (4.43) into (4.41) and taking into account the fact that at large values of $\Psi$ the coefficient $\widetilde{U}$ on the right-hand side of (4.41) is given by (4.29), we arrive at the following equation for the function $h\left(X_{*}\right)$ :

$$
\frac{h^{\prime}}{h}=\frac{1-\mathrm{e}^{X_{*}}}{1-\mathrm{e}^{X_{*}}} .
$$

The solution of this equation, satisfying the initial condition (4.44), is written as

$$
h\left(X_{*}\right)=\frac{2}{\mathrm{e}^{X_{*}}+2+\mathrm{e}^{-X_{*}}}, \quad X_{*} \in(-\infty, 0] .
$$


Let us now substitute (4.46) back into (4.43) and use for $\widetilde{H}$ its definition (4.40). Solving the resulting equation for $\widetilde{U}$, we find that near the outer edge of region $1 b$,

$$
\widetilde{U}=\sqrt{U_{e}^{2}+\frac{4 \mathrm{e}^{-\Psi}}{\mathrm{e}^{X_{*}}+2+\mathrm{e}^{-X_{*}}}}+\cdots, \quad \text { as } \quad \Psi \rightarrow \infty, \quad X_{*} \in(-\infty, 0] .
$$

In particular, if $X_{*} \rightarrow 0^{-}$, then it follows from (4.29) that

$$
U_{e}=\frac{1}{2}\left(-X_{*}\right)+\cdots,
$$

and (4.47) reduces to

$$
\widetilde{U}=\sqrt{\frac{1}{4}\left(-X_{*}\right)^{2}+\mathrm{e}^{-\Psi}}+\cdots, \quad \text { as } \quad \Psi \rightarrow \infty, \quad X_{*} \rightarrow 0^{-} .
$$

The asymptotic formula (4.49) has been obtained using a double-limit procedure. We first assumed that $\Psi$ tends to infinity, with $X_{*}$ remaining an order-one quantity; this led to (4.47). Then it was assumed that $X_{*}$ tends to zero. Therefore, strictly speaking, (4.49) remains valid provided that the first term in the square root is much larger than the second one, and a new region (let us call it region $1 b^{\prime}$ ) should be introduced where $\mathrm{e}^{-\Psi} /\left(-X_{*}\right)^{2}$ becomes an order-one quantity. A characteristic independent variable, $\eta$, in this region should satisfy the condition

$$
\frac{\mathrm{e}^{-\Psi}}{\left(-X_{*}\right)^{2}}=\phi(\eta)=O(1), \quad \text { as } \quad \Psi \rightarrow \infty, \quad X_{*} \rightarrow 0^{-} .
$$

Here $\phi$ is an arbitrary function of $\eta$. It is convenient to choose $\phi(\eta)=\mathrm{e}^{-\eta}$, and then we will have

$$
\eta=2 \ln \left(-X_{*}\right)+\Psi
$$

Using (4.51) in (4.49), we find that on approach to region $1 b^{\prime}$,

$$
\widetilde{U}=\left(-X_{*}\right) \sqrt{\frac{1}{4}+\mathrm{e}^{-\eta}}+\cdots .
$$

This suggests that the solution in region $1 b^{\prime}$ can be sought in the form

$$
\widetilde{U}=\left(-X_{*}\right) F(\eta)+\cdots, \quad \text { as } \quad X_{*} \rightarrow 0^{-},
$$

where the function $F(\eta)$ is such that

$$
F(\eta)=\sqrt{\frac{1}{4}+\mathrm{e}^{-\eta}}+\cdots, \quad \text { as } \quad \eta \rightarrow \infty .
$$

In order to find the function $F(\eta)$ we need to substitute (4.53), (4.51) and (4.48) into the momentum equation $(4.33 a)$. The three terms in this equation are calculated as

$$
\begin{gathered}
\widetilde{U} \frac{\partial \widetilde{U}}{\partial X_{*}}=-\left(-X_{*}\right)\left(2 F F^{\prime}+F^{2}\right), \quad U_{e} \frac{\mathrm{d} U_{e}}{\mathrm{~d} X_{*}}=-\frac{1}{4}\left(-X_{*}\right), \\
\widetilde{U} \frac{\partial}{\partial \Psi}\left(\widetilde{U} \frac{\partial \widetilde{U}}{\partial \Psi}\right)=\left(-X_{*}\right)^{3} F\left(F F^{\prime}\right)^{\prime} .
\end{gathered}
$$

We see that as $X_{*} \rightarrow 0^{-}$, the viscous term can be disregarded, which means that the flow in region $1 b^{\prime}$ appears to be effectively inviscid, and the equation for $F(\eta)$ assumes the form

$$
2 F F^{\prime}+F^{2}-\frac{1}{4}=0 .
$$


Its solution, satisfying the initial condition (4.54), is easily found to be (see Elliott, Smith \& Cowley 1983)

$$
F(\eta)=\sqrt{\frac{1}{4}+\mathrm{e}^{-\eta}} .
$$

Hence, the longitudinal velocity (4.53) in region $1 b^{\prime}$ is written as

$$
\widetilde{U}=\left(-X_{*}\right) \sqrt{\frac{1}{4}+\mathrm{e}^{-\eta}}+\cdots, \quad \text { as } \quad X_{*} \rightarrow 0^{-} .
$$

Substituting (4.51) into (4.59), we see that (4.49) can be used not only at the outer edge of region $1 b^{\prime}$ but also everywhere inside this region.

To complete the flow analysis in region $1 b$ we shall, finally, consider the limit

$$
X_{*} \rightarrow 0^{-}, \quad \Psi=O(1) .
$$

Since the velocity $U_{e}\left(X_{*}\right)$ at the outer edge of region $1 b$ is regular for all $X_{*}<0$, the asymptotic solution of $(4.33 a)$ with respect to the limit (4.60) can be sought in the form of the Taylor expansion

$$
\widetilde{U}\left(X_{*}, \Psi\right)=\widetilde{U}_{0}(\Psi)+\left(-X_{*}\right) \widetilde{U}_{1}(\Psi)+\cdots .
$$

Substitution of (4.61) and (4.48) into (4.33a) yields

$$
\widetilde{U}_{1}=-\frac{1}{2} \frac{\mathrm{d}^{2}}{\mathrm{~d} \Psi^{2}}\left(\widetilde{U}_{0}^{2}\right) .
$$

Matching of (4.61) with (4.59) shows that

$$
\widetilde{U}_{0}(\Psi)=\mathrm{e}^{-\Psi / 2}+\cdots, \quad \text { as } \quad \Psi \rightarrow \infty .
$$

If we now substitute (4.63) into (4.62), we find that

$$
\widetilde{U}_{1}(\Psi)=-\frac{1}{2} \mathrm{e}^{-\Psi}+\cdots, \quad \text { as } \quad \Psi \rightarrow \infty .
$$

\subsection{Displacement effect of region $1 b$}

The displacement effect of region $1 b$ is characterised by the angle the streamlines in this region make with the body surface. Integrating the continuity equation $(4.33 b)$ with the initial condition $(4.33 c)$, we have

$$
\frac{\widetilde{V}}{\widetilde{U}}=\frac{\partial}{\partial X_{*}} \int_{0}^{\Psi} \frac{\mathrm{d} \psi}{\widetilde{U}\left(X_{*}, \psi\right)} .
$$

Corresponding to the two limits, (4.50) and (4.60), which had to be used to describe the asymptotic behaviour of $\widetilde{U}$ in region $1 b$, we shall subdivide the integration interval in (4.65) into two parts,

$$
\psi \in\left[0, \Psi_{\Delta}\right] \text { and } \psi \in\left[\Psi_{\Delta}, \Psi\right],
$$

where

$$
\Psi_{\Delta}=-2 \ln \left(-X_{*}\right)-\Delta,
$$

with $\Delta$ being such that

$$
1 \ll \Delta \ll-2 \ln \left(-X_{*}\right) .
$$

In the first interval, we have to use the asymptotic expansion (4.61). This gives

$$
\frac{\partial}{\partial X_{*}} \int_{0}^{\psi_{\Delta}} \frac{\mathrm{d} \psi}{\widetilde{U}\left(X_{*}, \psi\right)}=-\int_{0}^{\psi_{\Delta}} \frac{1}{\widetilde{U}^{2}} \frac{\partial \widetilde{U}}{\partial X_{*}} \mathrm{~d} \psi=\int_{0}^{\psi_{\Delta}} \frac{\widetilde{U}_{1}(\psi)}{\left[\widetilde{U}_{0}(\psi)\right]^{2}} \mathrm{~d} \psi
$$


which, in view of (4.63) and (4.64), may be approximated as

$$
\begin{array}{r}
\int_{0}^{\Psi_{\Delta}} \frac{\widetilde{U}_{1}(\psi)}{\left[\widetilde{U}_{0}(\psi)\right]^{2}} \mathrm{~d} \psi=\int_{0}^{-2 \ln \left(-X_{*}\right)-\Delta}\left\{\frac{\widetilde{U}_{1}(\psi)}{\left[\widetilde{U}_{0}(\psi)\right]^{2}}+\frac{1}{2}\right\} \mathrm{d} \psi+\ln \left(-X_{*}\right)+\frac{1}{2} \Delta \\
=\ln \left(-X_{*}\right)+\frac{1}{2} \Delta+D+\cdots, \text { as } X_{*} \rightarrow 0^{-},
\end{array}
$$

with the constant $D$ given by

$$
D=\int_{0}^{\infty}\left\{\frac{\widetilde{U}_{1}(\psi)}{\left[\widetilde{U}_{0}(\psi)\right]^{2}}+\frac{1}{2}\right\} \mathrm{d} \psi
$$

Now we turn to the second interval in (4.66), where $\widetilde{U}$ is represented by (4.59). We have

$$
\begin{aligned}
\frac{\partial}{\partial X_{*}} \int_{\Psi_{\Delta}}^{\Psi} \frac{\mathrm{d} \psi}{\widetilde{U}\left(X_{*}, \psi\right)} & =\frac{1}{\left(-X_{*}\right)^{2}} \int_{-\Delta}^{2 \ln \left(-X_{*}\right)+\Psi}\left(\frac{1}{F}+2 \frac{F^{\prime}}{F^{2}}\right) \mathrm{d} \eta \\
& =\frac{2}{\left(-X_{*}\right)^{2}}\left[\ln \frac{\sqrt{\frac{1}{4}+\mathrm{e}^{-\eta}}+\frac{1}{2}}{\sqrt{\frac{1}{4}+\mathrm{e}^{-\eta}}-\frac{1}{2}}-\frac{1}{\sqrt{\frac{1}{4}+\mathrm{e}^{-\eta}}}\right]_{-\Delta}^{2 \ln \left(-X_{*}\right)+\Psi} \\
& =\frac{2}{\left(-X_{*}\right)^{2}}\left[\Psi+2 \ln \left(-X_{*}\right)-2\right]+\cdots
\end{aligned}
$$

Comparing (4.72) with (4.70) it is easy to see that the displacement effect of region $1 b$ is predominantly due to the contribution from region $1 b^{\prime}$. Using (4.72) on the righthand side of (4.65) and expressing the left-hand side in terms of original variables, $U$ and $V$, as defined by (4.30), we arrive at a conclusion that at the outer edge of the viscous sublayer (region $1 b$ )

$$
\frac{V}{U}=\frac{2 \Psi}{\left(-X_{*}\right)^{2}}+\frac{4}{\left(-X_{*}\right)^{2}}\left[\ln \left(-X_{*}\right)-1\right]+\cdots .
$$

Let us now perform the matching of the streamline slope, $V / U$, in regions $1 a$ and $1 b$. Everywhere in region $1 a$ it is given by (4.14), with $U_{*}$ and $V_{*}$ being defined by (4.8). Hence, we have

$$
\frac{V}{U}=U_{w}^{2}\left(\frac{1}{\sqrt{1-2 P_{*}}}-\frac{1}{\sqrt{2 \Psi_{*}+1-2 P_{*}}}\right) \frac{\mathrm{d} P_{*}}{\mathrm{~d} X_{*}} .
$$

At the 'bottom' of region $1 a$, where $\Psi_{*}$ is small, (4.74) reduces to

$$
\frac{V}{U}=U_{w}^{2} \frac{\Psi_{*}}{\left(1-2 P_{*}\right)^{3 / 2}} \frac{\mathrm{d} P_{*}}{\mathrm{~d} X_{*}}+\cdots .
$$

Recall that the pressure acting on the boundary layer is a known function given by (4.28). For small values of $X_{*}$ it may be represented using the Taylor expansion

$$
P_{*}=\frac{1}{2}-\frac{1}{8}\left(-X_{*}\right)^{2}+\cdots, \quad \text { as } \quad X_{*} \rightarrow 0^{-} .
$$

Substituting (4.76) into (4.75), we can conclude that at the bottom of region $1 a$,

$$
\frac{V}{U}=\frac{2 U_{w}^{2} \Psi_{*}}{\left(-X_{*}\right)^{2}}+\cdots \text {. }
$$


It is easily seen that (4.77) reproduces the first term in (4.73). Hence, the second term should be solely attributed to the displacement effect of region $1 b$,

$$
\left.\frac{V}{U}\right|_{\text {region } 1 b}=\frac{4}{\left(-X_{*}\right)^{2}}\left[\ln \left(-X_{*}\right)-1\right]+\cdots, \quad \text { as } \quad X_{*} \rightarrow 0^{-} .
$$

Let us now return to (4.74), which describes the displacement effect of region $1 a$, and rearrange it in the form

$$
\frac{V}{U}=\frac{U_{w}^{2}}{\sqrt{1-2 P_{*}}}\left(1-\frac{1}{\sqrt{1+2 \xi}}\right) \frac{\mathrm{d} P_{*}}{\mathrm{~d} X_{*}},
$$

with

$$
\xi=\frac{\Psi_{*}}{1-2 P_{*}}
$$

It follows from $(4.79 a)$ that in the limit as $X_{*} \rightarrow 0^{-}$, the displacement of region $1 a$ is predominantly due to region $1 a^{\prime}$, where $\xi=O(1)$. Indeed, setting $\xi=0$ in $(4.79 a)$ results in $V / U=0$, and when $\xi$ becomes large as compared to one, the streamline slope reaches its limiting value

$$
\left.\frac{V}{U}\right|_{\text {region } 1 a}=\frac{U_{w}^{2}}{\sqrt{1-2 P_{*}}} \frac{\mathrm{d} P_{*}}{\mathrm{~d} X_{*}} \cdots=\frac{1}{2} U_{w}^{2}+\cdots \quad \text { as } \quad X_{*} \rightarrow 0^{-} .
$$

Comparing (4.80) with (4.78), and keeping in mind that $U_{w} \gg 1$, one can see that as long as $X_{*}$ is not too small, the displacement effect of region $1 a$ is dominant, and the analysis leading to (4.15) remains valid. However, for any $U_{w}$, no matter how large, there exists a vicinity of the singular point $X_{*}=0$, where the displacement effect of region $1 b$ can no longer be ignored. Our task now will be to examine the flow behaviour in the vicinity of this region.

\subsection{Composite solution}

In the region in question (we shall call it region $C$ ) we introduce a new longitudinal coordinate, $\breve{X}$, such that

$$
X_{*}=\sigma \breve{X}
$$

where the scaling parameter $\sigma$ is defined by

$$
\frac{2|\ln \sigma|}{\sigma^{2}}=U_{w}^{2}
$$

This ensures that the streamline angle (4.78) produced by region $1 b$ is of the same order as the streamline angle (4.80) produced by region $1 a$.

Region $C$ is composed of the stream filaments that pass through regions $1 a^{\prime}$ and $1 b^{\prime}$. The longitudinal velocity in region $1 a^{\prime}$ is given by (4.13). Substituting (4.76) into (4.13), we find

$$
U_{*}=\frac{1}{2}\left(-X_{*}\right) \sqrt{1+2 \xi}+\cdots, \quad \xi=\frac{4 \Psi_{*}}{\left(-X_{*}\right)^{2}} .
$$


If we now use (4.81) in (4.83) and (4.76), and return to the original variables (4.8), then we will see that in region $1 a^{\prime}$,

$$
\begin{aligned}
U & =U_{w} \sigma \frac{1}{2}(-\breve{X}) \sqrt{1+2 \xi}+\cdots, \quad \xi=\frac{4 \Psi}{U_{w}^{2} \sigma^{2}(-\breve{X})^{2}}, \\
P & =\frac{1}{2} U_{w}^{2}-U_{w}^{2} \sigma^{2} \frac{1}{8}(-\breve{X})^{2}+\cdots .
\end{aligned}
$$

This suggests that the solution in region $C$ has to be sought in the form

$$
\begin{gathered}
U=U_{w} \sigma \breve{U}+\cdots, \quad P=\frac{1}{2} U_{w}^{2}+U_{w}^{2} \sigma^{2} \breve{P}+\cdots, \\
X=\frac{\sigma}{U_{w}} \breve{X}, \quad \Psi=U_{w}^{2} \sigma^{2} \breve{\Psi},
\end{gathered}
$$

with functions $\breve{U}(\breve{X}, \breve{\Psi})$ and $\breve{P}(\breve{X}, \breve{\Psi})$ satisfying the following matching condition with the solution in region $1 a^{\prime}$ :

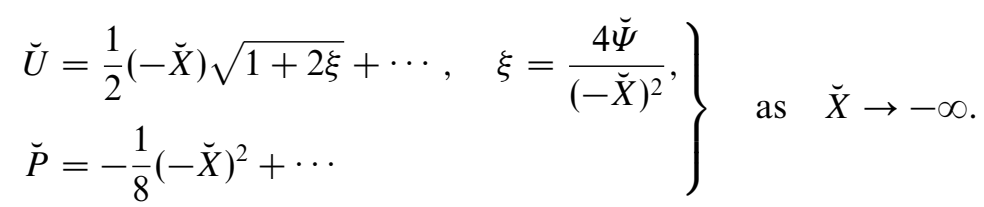

In order to formulate the matching condition with the solution in region $1 b^{\prime}$, we need to return to (4.59). Combining it with the first of equations (4.30) and applying the rescaling (4.81) renders the solution in region $1 b^{\prime}$ in the form

$$
U=U_{w} \sigma(-\breve{X}) \sqrt{\frac{1}{4}+\mathrm{e}^{-\eta}}+\cdots
$$

which means that the matching condition sought is

$$
\breve{U}=(-\breve{X}) \sqrt{\frac{1}{4}+\mathrm{e}^{-\eta}}+\cdots, \quad \text { as } \quad \breve{X} \rightarrow-\infty .
$$

Here the similarity variable $\eta$ is defined by (4.51). We now need to express it in terms of the variables $(4.86 b)$ of region $C$. Substituting (4.81) and the second equation in (4.86) into (4.51), we find

$$
\eta=2 \ln \sigma+2 \ln (-\breve{X})+U_{w}^{2} \sigma^{2} \breve{\Psi} .
$$

Finally, it follows from (4.82) that $2 \ln \sigma=-U_{w}^{2} \sigma^{2}$, and therefore, we can express the above equation in the form

$$
\eta=2 \ln (-\breve{X})+\chi(\breve{\Psi}-1)
$$

where $\chi=2|\ln \sigma|$.

The boundary condition (4.89) and (4.91) clearly shows that the function $\breve{U}$ depends not only on the coordinates $\breve{X}$ and $\breve{\Psi}$ but also on a large parameter $\chi$. Hence, the representations of $U$ and $P$ in the solution (4.86) for region $C$ cannot be thought of as Poincaré-type straightforward asymptotic expansions. This is an unusual situation, and it arises due to the fact that region $C$ lies in the overlap region between regions $1 a^{\prime}$ and $1 b^{\prime}$. A traditional way of dealing with situations like these is to use composite asymptotic expansions.

Most conveniently, it may be done by using the fact that the flow is inviscid both in regions $1 a^{\prime}$ and $1 b^{\prime}$. Therefore, it is expected to be inviscid also in region $C$, in which case the momentum equation (4.1a) reduces to the Bernoulli equation

$$
\frac{1}{2} U^{2}+P=H(\Psi) \text {. }
$$


It follows from (4.86) that in region $C$ the Bernoulli function $H(\Psi)$ may be written in the form

where

$$
H=\frac{1}{2} U_{w}^{2}+U_{w}^{2} \sigma^{2} \breve{H}(\breve{\Psi}),
$$

$$
\breve{H}(\breve{\Psi})=\frac{1}{2} \breve{U}^{2}+\breve{P} .
$$

The function $\breve{H}(\breve{\Psi})$ does not change along streamlines, and can be found from the solutions in regions $1 a^{\prime}$ and $1 b^{\prime}$. Using (4.87) on the right-hand side of (4.94), we have

$$
\breve{H}_{1 a^{\prime}}=\breve{\Psi} .
$$

Similarly, it follows from (4.89) and (4.91) that

$$
\breve{H}_{1 b^{\prime}}=\frac{1}{2} \mathrm{e}^{-\chi(\breve{\Psi}-1)} .
$$

The composite Bernoulli function is obtained by adding (4.95) and (4.96) together,

$$
\breve{H}=\breve{\Psi}+\frac{1}{2} \mathrm{e}^{-\chi(\breve{\Psi}-1)} .
$$

If we now substitute (4.97) back into (4.94), and solve the resulting equation for $\breve{U}$, we will find that everywhere in region $C$,

$$
\breve{U}=\sqrt{2 \breve{\Psi}+\mathrm{e}^{-\chi(\breve{\Psi}-1)}-2 \breve{P}} .
$$

Here the pressure function, $\breve{P}(\breve{X})$, is still unknown. In order to find $\breve{P}(\breve{X})$, we need to use the interaction law (4.1c). The second term on the right-hand side of (4.1c) may be found by integrating the continuity equation $(4.1 b)$ with respect to $\Psi$,

$$
\frac{V}{U}=\frac{\partial}{\partial X} \int_{0}^{\Psi} \frac{\mathrm{d} \psi}{U(X, \psi)} .
$$

Expressing the right-hand side of (4.99) in terms of the variables (4.86) of region $C$, we have

$$
\frac{V}{U}=U_{w}^{2} \frac{\partial}{\partial \breve{X}} \int_{-\infty}^{\check{\Psi}} \frac{\mathrm{d} \breve{\psi}}{\breve{U}(\breve{X}, \breve{\psi})} .
$$

Note that the integration in (4.100) starts at the lower edge of region $C$. This is to account for the fact that region $C$ produces the dominant contribution to the displacement thickness of the boundary layer.

Let us now set $\breve{\Psi}=\infty$ in (4.100) and substitute it into (4.1c). In the leading-order approximation, the left-hand side of $(4.1 c)$ equals $\frac{1}{2} U_{w}^{2}$, as one can see from the representation (4.86) of $P$ in region $C$. Hence, we can write

$$
\frac{1}{2} U_{w}^{2}=U_{w}^{2} \frac{\partial}{\partial \breve{X}} \int_{-\infty}^{\infty} \frac{\mathrm{d} \breve{\Psi}}{\breve{U}(\breve{X}, \breve{\Psi})} .
$$

Here we have omitted the impinging shock term $P_{s} \mathscr{H}(X)$, aiming to solve the selfinduced separation problem first.

It remains to substitute (4.98) into (4.101), which leads to the following equation for $\breve{P}(\breve{X})$ :

$$
\frac{\mathrm{d} \breve{P}}{\mathrm{~d} \breve{X}} \int_{-\infty}^{\infty} \frac{\mathrm{d} \breve{\Psi}}{\left[2 \breve{\Psi}+\mathrm{e}^{-\chi(\breve{\Psi}-1)}-2 \breve{P}\right]^{3 / 2}}=\frac{1}{2}
$$




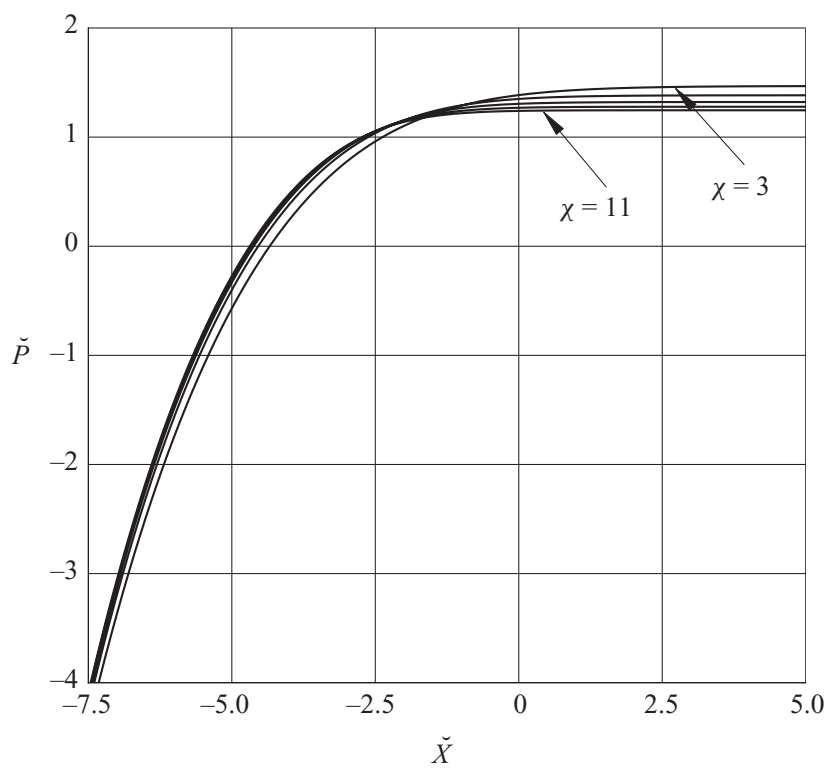

FIGURE 6. Pressure distribution in region $C$ for the values of parameter $\chi$ from 3.0 to 11.0 plotted with the interval $\Delta \chi=2.0$.

The initial condition for this equation is given by the matching condition (4.87),

$$
\breve{P}=-\frac{1}{8}(-\breve{X})^{2}+\cdots, \quad \text { as } \quad \breve{X} \rightarrow-\infty .
$$

The results of the numerical solution of the initial-value problem (4.102) are displayed in figure 6. An important point to note is that for any $\chi$ the function $\breve{P}(\breve{X})$ has a finite limit, $\breve{P}_{c}$, as $X \rightarrow \infty$. We shall now confirm this result analytically.

It follows from (4.98) that for all $\breve{X}$, the minimum of the velocity $\breve{U}$ is achieved on the streamline with

$$
\breve{\Psi}=\breve{\Psi}_{0}=1+\frac{1}{\chi} \ln \frac{\chi}{2},
$$

where the Bernoulli function (4.97) assumes its minimal value

$$
\breve{H}_{0}=1+\frac{1}{\chi}\left(1+\ln \frac{\chi}{2}\right) \text {. }
$$

It also follows from (4.98) that the pressure cannot exceed the value

$$
\breve{P}_{c}=\breve{H}_{0}=1+\frac{1}{\chi}\left(1+\ln \frac{\chi}{2}\right) \text {. }
$$

In the limit, as $\breve{P} \rightarrow \breve{P}_{c}$, the velocity $\breve{U}$ tends to zero on the streamline (4.103), but remains positive on other streamlines.

In order to determine how the pressure $\breve{P}$ approaches its critical value $\breve{P}_{c}$, it is convenient to express $(4.102 a)$ in the form

$$
\frac{\mathrm{d} \breve{P}}{\mathrm{~d} \breve{X}} \int_{-\infty}^{\infty} \frac{\mathrm{d} \breve{\Psi}}{\left[2\left(\breve{H}-\breve{H}_{0}\right)-2\left(\breve{P}-\breve{P}_{c}\right)\right]^{3 / 2}}=\frac{1}{2},
$$

which shows that for small $\left|\breve{P}-\breve{P}_{c}\right|$, the dominant contribution to the integral in (4.106) is given by a small vicinity of the point $\breve{\Psi}=\breve{\Psi}_{0}$, where the Bernoulli function 
(4.97) may be approximated by the Taylor expansion

$$
\breve{H}=\breve{H}_{0}+\frac{1}{2} \chi\left(\breve{\Psi}-\breve{\Psi}_{0}\right)^{2}+\cdots .
$$

We have

$$
\int_{-\infty}^{\infty} \frac{\mathrm{d} \breve{\Psi}}{\left[2\left(\breve{H}-\breve{H}_{0}\right)-2\left(\breve{P}-\breve{P}_{c}\right)\right]^{3 / 2}} \approx \int_{\breve{\Psi}_{0}-\Delta}^{\breve{\Psi}_{0}+\Delta} \frac{\mathrm{d} \breve{\Psi}}{\left[\chi\left(\breve{\Psi}-\breve{\Psi}_{0}\right)^{2}+2\left(\breve{P}_{c}-\breve{P}\right)\right]^{3 / 2}}
$$

Here $\Delta$ has to be chosen such that

$$
\sqrt{\left(\breve{P}_{c}-\breve{P}\right) / \chi} \ll \Delta \ll 1 .
$$

The integral on the right-hand side of (4.108) may be calculated analytically,

$$
\int_{\breve{\Psi}_{0}-\Delta}^{\breve{\Psi}_{0}+\Delta} \frac{\mathrm{d} \breve{\Psi}}{\left[\chi\left(\breve{\Psi}-\breve{\Psi}_{0}\right)^{2}+2\left(\breve{P}_{c}-\breve{P}\right)\right]^{3 / 2}}=\frac{1}{\sqrt{\chi}\left(\breve{P}_{c}-\breve{P}\right)},
$$

which allows (4.106) to be written in the form

$$
\frac{\mathrm{d} \breve{P}}{\mathrm{~d} \breve{X}}=\frac{\sqrt{\chi}}{2}\left(\breve{P}_{c}-\breve{P}\right)
$$

The general solution of (4.111) is written as

$$
\breve{P}=\breve{P}_{c}-\exp \left[-\frac{1}{2} \sqrt{\chi}\left(\breve{X}-\breve{X}_{0}\right)\right]+\cdots, \quad \text { as } \quad \breve{X} \rightarrow \infty,
$$

where $\breve{X}_{0}$ stands for the constant of integration.

The corresponding formula for the longitudinal velocity, $\breve{U}$, may be now obtained from the Bernoulli equation. Writing (4.98) in the form

$$
\breve{U}=\sqrt{2\left(\breve{H}-\breve{H}_{0}\right)-2\left(\breve{P}-\breve{P}_{c}\right)}
$$

and substituting (4.107) and (4.112) into (4.113), we find that

$$
\breve{U}=\exp \left[-\frac{1}{4} \sqrt{\chi}\left(\breve{X}-\breve{X}_{0}\right)\right] \sqrt{2+\xi^{2}}+\cdots, \quad \text { as } \quad \breve{X} \rightarrow \infty,
$$

where

$$
\xi=\sqrt{\chi} \frac{\breve{\Psi}-\breve{\Psi}_{0}}{\exp \left[-(1 / 4) \sqrt{\chi}\left(\breve{X}-\breve{X}_{0}\right)\right]} .
$$

The results of the flow analysis in region $C$ may be summarised as follows. The pressure in region $C$ experiences a monotonic rise. It does not lead to the formation of a flow reversal, characteristic of the separation process, but it does lead to a rather fast deceleration of the fluid, as described by (4.114). When the fluid velocity becomes small enough, the forces of internal viscosity can no longer be disregarded, which means that in order to complete the flow analysis, one more region (we shall call it region $V$ ), which lies behind region $C$, has to be considered.

\subsection{Viscous region $V$}

The viscous region forms where the convective term in the momentum equation $(4.1 a)$ becomes comparable with the viscous term, i.e.

$$
U \frac{\partial U}{\partial X} \sim U \frac{\partial}{\partial \Psi}\left(U \frac{\partial U}{\partial \Psi}\right)
$$


More conveniently, this requirement may be written as

$$
\frac{\partial U}{\partial X} \sim \frac{\partial^{2}}{\partial \Psi^{2}}\left(\frac{U^{2}}{2}\right)
$$

It follows from (4.114) and (4.86) that on approaching the viscous region, the longitudinal velocity component may be expressed in the form

$$
\begin{gathered}
U=\sqrt{\chi} \exp \left[-\frac{1}{4} \sqrt{\chi}\left(\breve{X}-\breve{X}_{0}\right)\right] \sqrt{2+\xi^{2}}+\cdots, \quad \xi=\sqrt{\chi} \frac{\breve{\Psi}-\breve{\Psi}_{0}}{\exp \left[-\frac{1}{4} \sqrt{\chi}\left(\breve{X}-\breve{X}_{0}\right)\right]}, \\
X=\frac{\sqrt{\chi}}{U_{w}^{2}} \breve{X}, \quad \Psi=\chi \breve{\Psi} .
\end{gathered}
$$

When calculating the scaling coefficients in (4.117), we have used the small parameter $\sigma$ as defined by (4.81) and the large parameter $\chi=2|\ln \sigma|$.

Differentiation of (4.117) results in

$$
\frac{\partial U}{\partial X}=-\frac{1}{2} U_{w}^{2} \sqrt{\chi} \exp \left[-\frac{1}{4} \sqrt{\chi}\left(\breve{X}-\breve{X}_{0}\right)\right] \frac{1}{\sqrt{2+\xi^{2}}}, \quad \frac{\partial^{2}}{\partial \Psi^{2}}\left(\frac{U^{2}}{2}\right)=1 .
$$

Keeping in mind that in the region of interest $\xi=O(1)$, we can conclude that the two terms in (4.116) are in balance with each other provided that

$$
U_{w}^{2} \sqrt{\chi} \exp \left[-\frac{1}{4} \sqrt{\chi}\left(\breve{X}-\breve{X}_{0}\right)\right]=\Lambda
$$

where $\Lambda$ is an order-one quantity. Solving (4.119) for $\breve{X}$, we find

$$
\breve{X}=\breve{X}_{0}+\frac{4}{\sqrt{\chi}} \ln \left(U_{w}^{2} \sqrt{\chi}\right)-\frac{4}{\sqrt{\chi}} \ln \Lambda .
$$

This shows that in the viscous region $V$, the characteristic longitudinal coordinate, $\tilde{x}$, should be introduced through the equation

$$
\breve{X}=\breve{X}_{0}+\frac{4}{\sqrt{\chi}} \ln \left(U_{w}^{2} \sqrt{\chi}\right)+\frac{\tilde{x}}{\sqrt{\chi}} .
$$

It further follows from (4.119) and the equation for $\xi$ in (4.117) that in region $V$ the streamfunction should be written as

$$
\breve{\Psi}=\breve{\Psi}_{0}+\frac{\tilde{\psi}}{U_{w}^{2} \chi},
$$

where $\tilde{\psi}$ is an order-one quantity. Similarly, one can deduce from (4.112) and (4.114a) that

$$
\breve{P}=\breve{P}_{c}+\frac{\tilde{p}}{U_{w}^{4} \chi}+\cdots, \quad \breve{U}=\frac{\tilde{u}}{U_{w}^{2} \sqrt{\chi}}+\cdots .
$$

It remains to express (4.121), (4.122) and (4.123) in terms of the original variables (4.86), and we can conclude that in region $V$ the solution of the interaction problem (4.1) has to be sought in the form

$$
\left.\begin{array}{c}
U=\frac{1}{U_{w}^{2}} \tilde{u}+\cdots, \quad V=\tilde{v}+\cdots, \quad P=\frac{1}{2} U_{w}^{2}+\chi \breve{P}_{c}+\frac{1}{U_{w}^{4}} \tilde{p}+\cdots, \\
X=\frac{\sqrt{\chi}}{U_{w}^{2}} \breve{X}_{0}+\frac{4}{U_{w}^{2}} \ln \left(U_{w}^{2} \sqrt{\chi}\right)+\frac{1}{U_{w}^{2}} \tilde{x}, \quad \Psi=\chi \breve{\Psi}_{0}+\frac{1}{U_{w}^{2}} \tilde{\psi} .
\end{array}\right\}
$$


Here the order of magnitude of the lateral velocity component, $V$, is found as usual through making use of the continuity equation $(4.1 b)$.

Substitution of $(4.124)$ into $(4.1 a)-(4.1 c)$ results in the following set of equations for the functions $\tilde{u}(\tilde{x}, \tilde{\psi}), \tilde{v}(\tilde{x}, \tilde{\psi})$ and $\tilde{p}(\tilde{x}, \tilde{\psi})$ :

$$
\begin{aligned}
\tilde{u} \frac{\partial \tilde{u}}{\partial \tilde{x}}+\frac{\mathrm{d} \tilde{p}}{\mathrm{~d} \tilde{x}} & =\tilde{u} \frac{\partial}{\partial \tilde{\psi}}\left(\tilde{u} \frac{\partial \tilde{u}}{\partial \tilde{\psi}}\right), \\
\frac{\partial}{\partial \tilde{\psi}}\left(\frac{\tilde{v}}{\tilde{u}}\right) & =\frac{\partial}{\partial \tilde{x}}\left(\frac{1}{\tilde{u}}\right), \\
\lim _{\tilde{\psi} \rightarrow \infty} \frac{\tilde{v}}{\tilde{u}} & =\frac{1}{2} .
\end{aligned}
$$

Equation $(4.125 a)$ is parabolic and requires an initial condition for the function $\tilde{u}(\tilde{x}, \tilde{\psi})$. The latter is deduced from matching with the solution (4.117) in region $C$. We have

$$
\tilde{u}=\mathrm{e}^{-\tilde{x} / 4} \sqrt{2+\frac{\tilde{\psi}^{2}}{\mathrm{e}^{-\tilde{x} / 2}}}+\cdots, \quad \text { as } \quad \tilde{x} \rightarrow-\infty .
$$

To close the problem, we also need a boundary condition for the continuity equation (4.125b). Since region $V$ gives the dominant contribution to the displacement effect of the boundary layer, we can write

$$
\left.\frac{\tilde{v}}{\tilde{u}}\right|_{\tilde{\psi}=-\infty}=0 .
$$

The boundary-value problem (4.125)-(4.127) is not new. Earlier, it has been encountered in theoretical studies of high-Reynolds-number flows on a number of occasions. Sychev (1978) was the first to formulate this problem in the course of his analysis of viscous wake breakdown in incompressible fluid flow. It was then shown to play a central role in describing the incompressible boundary-layer separation on a downstream moving wall (see Sychev 1979, 1984). Later, Battam et al. (2004) demonstrated that this problem also describes the formation of the flow reversal in the viscous wake interacting with a shock wave in supersonic gas flows. Interestingly enough, this problem admits an analytical solution which was first published by Sychev (1987). We shall now give a short account of Sychev's arguments.

Equations $(4.125 a)$ and $(4.125 b)$ are the boundary-layer momentum and continuity equations written in the von Mises variables. Unlike in classical Prandtl's formulation, the pressure, $\tilde{p}(\tilde{x})$, in $(4.125 a)$ is not known in advance. It has to be found as a part of the solution of the problem (4.125)-(4.127) together with the velocity components $\tilde{u}(\tilde{x}, \tilde{\psi}), \tilde{v}(\tilde{x}, \tilde{\psi})$. Recall that the independent variables, $(\tilde{x}, \tilde{\psi})$, are introduced through (4.124); here $\tilde{x}$ is an appropriately scaled longitudinal coordinate measured along the plate surface, and $\tilde{\psi}$ is the streamfunction measured from the 'dividing streamline', $\Psi=\chi \breve{\Psi}_{0}$, where $\tilde{\psi}=0$. It is interesting to note that $(4.125 a)$ is invariant with respect to the transformation $\tilde{\psi} \rightarrow-\tilde{\psi}$, and so is the initial condition (4.126). This suggests that the solution for the longitudinal velocity component, $\tilde{u}(\tilde{x}, \tilde{\psi})$, has to be symmetric with respect to the 'dividing streamline', $\tilde{\psi}=0$, and the 'dividing streamline' has to be the locus of minimal longitudinal velocity everywhere in region $V$.

Equations $(4.125 a)$ and $(4.125 b)$ may also be written in the conventional form using curvilinear orthogonal coordinates $(\tilde{s}, \tilde{n})$ as the independent variable. For our purpose, it is convenient to choose $\tilde{s}$ to be the arclength along the 'dividing streamline', and $\tilde{n}$ the distance measured perpendicular to this streamline. In the boundary-layer 
approximation, the curvilinear coordinates $(\tilde{s}, \tilde{n})$ are related to the von Mises variables $(\tilde{x}, \tilde{\psi})$ as

$$
\tilde{s}=\tilde{x}, \quad \tilde{n}=\int_{0}^{\tilde{\psi}} \frac{\mathrm{d} \tilde{\psi}^{\prime}}{\tilde{u}\left(\tilde{x}, \tilde{\psi}^{\prime}\right)} .
$$

Treating the streamfunction $\tilde{\psi}$ as a function of $\tilde{s}$ and $\tilde{n}$, and pressure $\tilde{p}$ as a function of $\tilde{s}$, we can express the boundary-value problem $(4.125)-(4.127)$ in the form (see the Appendix)

$$
\begin{array}{ll}
\frac{\partial \tilde{\psi}}{\partial \tilde{n}} \frac{\partial^{2} \tilde{\psi}}{\partial \tilde{s} \partial \tilde{n}}-\frac{\partial \tilde{\psi}}{\partial \tilde{s}} \frac{\partial^{2} \tilde{\psi}}{\partial \tilde{n}^{2}}=-\frac{\mathrm{d} \tilde{p}}{\mathrm{~d} \tilde{s}}+\frac{\partial^{3} \tilde{\psi}}{\partial \tilde{n}^{3}}, \\
\tilde{\psi}=\sqrt{2} \mathrm{e}^{-\tilde{s} / 4} \sinh \tilde{n}+\cdots, & \text { as } \quad \tilde{s} \rightarrow-\infty, \\
\tilde{\psi}=\frac{\partial^{2} \tilde{\psi}}{\partial \tilde{n}^{2}}=0, & \text { at } \quad \tilde{n}=0, \\
\frac{\partial \tilde{\psi} / \partial \tilde{s}}{\partial \tilde{\psi} / \partial \tilde{n}}=-\frac{1}{4}, & \text { at } \quad \tilde{n}=\infty .
\end{array}
$$

Here, thanks to the symmetry of the flow, we restrict our attention to the flow field above the 'dividing streamline' $(\tilde{n}>0)$. The symmetry conditions are expressed by $(4.129 c)$.

By direct substitution it is easily verified that the solution to (4.129) has the form

$$
\tilde{\psi}=\sqrt{2} \mathrm{e}^{-\tilde{s} / 4} \sinh \tilde{n}-4 \tilde{n}, \quad \tilde{p}=-\mathrm{e}^{-\tilde{s} / 2} .
$$

The longitudinal velocity is calculated as

$$
\tilde{u}=\frac{\partial \tilde{\psi}}{\partial \tilde{n}}=\sqrt{2} \mathrm{e}^{-\tilde{s} / 2} \cosh \tilde{n}-4 .
$$

We see that the fluid velocity first turns zero at the point on the 'dividing streamline' $(\tilde{n}=0)$, where $\tilde{s}=-6 \ln 2$. Downstream of this point, two eddies (which are symmetric with respect to 'dividing streamline') form in the flow.

\section{Conclusions}

In this paper, the process of the shock wave interaction with the boundary layer has been investigated assuming that the flow outside the boundary layer is supersonic and the point at which the shock impinges upon the boundary layer moves upstream along the boundary layer with the speed $V_{s h}$. The flow was studied based on the asymptotic analysis of the Navier-Stokes equations at large values of the Reynolds number, $R e$. Two flow regimes were considered. In the first regime, the shock speed $V_{\text {sh }}$ was assumed to be an order $R e^{-1 / 8}$ quantity. In this case, the interaction between the boundary layer and shock wave is described by the 'triple-deck theory'. The 'tripledeck equations' have been calculated before by various authors for the motionless shock. Our task was to see what happens when the shock moves with respect to the body surface in the upstream direction. We found that the shock motion suppresses the boundary-layer separation. In fact, the higher the shock speed, $V_{s h}$, the larger the pressure jump in the shock is required to cause the formation of a recirculation region in the boundary layer. We also found that instead of a single recirculation region, which is normally observed in the case of the motionless shock, two eddies sitting on top of each other form in the boundary layer exposed to a moving shock. 
In the second part of the paper, we re-examined the flow under the assumption that $V_{s h} \gg R e^{-1 / 8}$. We found that in this flow regime, the entire analysis of the fluid motion could be conducted analytically. We deduced the corresponding equations of motion starting with the 'triple-deck' equations assuming that $V_{s h} / R e^{-1 / 8} \rightarrow \infty$. Alternatively, the analysis could be performed starting with the Navier-Stokes equations and assuming that $1 \gg V_{s h} \gg R e^{-1 / 8}$ (see Araki 2006). The resulting asymptotic equations are, of course, the same; this may be viewed as one more confirmation of the validity of Kaplun's (1967) extension theorem.

Our main goal was to generalise the Moore-Rott-Sears condition for the flow considered. It is known that in unsteady flows the formation of a flow reversal does not necessarily mean that the boundary-layer separation takes place. The separation has to be identified by a singularity in the solution for the viscous sublayer that forms near the body surface. In the flow considered, the singularity was found to appear at the outer edge of the sublayer. This situation is rather unusual, and it makes the Poincar-type straightforward asymptotic expansions inapplicable. Instead, composite asymptotic expansions had to be used in a part of the flow field. Still, similar to the incompressible flow case (see Sychev 1987), the formation of the recirculation region was shown to be a viscous process, taking place in a region where the displacement thickness of the boundary is known to grow linearly with distance along the body surface. The solution to the corresponding boundary-value problem is written a simple analytic form.

\section{Appendix. Inverse von Mises transformation}

The boundary-value problem (4.125)-(4.127) is transformed to the form (4.129) in the following way. Given the function $\tilde{u}(\tilde{x}, \tilde{\psi})$, we introduce new independent variables

such that

$$
\tilde{s}=\tilde{s}(\tilde{x}, \tilde{\psi}), \quad \tilde{n}=\tilde{n}(\tilde{x}, \tilde{\psi}),
$$

$$
\tilde{s}(\tilde{x}, \tilde{\psi})=\tilde{x}, \quad \tilde{n}(\tilde{x}, \tilde{\psi})=\int_{0}^{\tilde{\psi}} \frac{\mathrm{d} \tilde{\psi}^{\prime}}{\tilde{u}\left(\tilde{x}, \tilde{\psi}^{\prime}\right)} .
$$

Let us consider a pair of functions

$$
\tilde{x}=\tilde{x}(\tilde{s}, \tilde{n}), \quad \tilde{\psi}=\tilde{\psi}(\tilde{s}, \tilde{n}),
$$

inverse to (A 1). Substitution of (A 1) into the second equation in (A 3) leads to the identity

$$
\tilde{\psi} \equiv \tilde{\psi}[\tilde{s}(\tilde{x}, \tilde{\psi}), \tilde{n}(\tilde{x}, \tilde{\psi})] .
$$

Differentiation of (A 4) with respect to $\tilde{x}$ and $\tilde{\psi}$ gives

$$
0=\frac{\partial \tilde{\psi}}{\partial \tilde{s}}+\frac{\partial \tilde{\psi}}{\partial \tilde{n}} \frac{\partial \tilde{n}}{\partial \tilde{x}}, \quad 1=\frac{\partial \tilde{\psi}}{\partial \tilde{n}} \frac{1}{\tilde{u}} .
$$

It follows from the second equation in (A 5) that

$$
\frac{\partial \tilde{\psi}}{\partial \tilde{n}}=\tilde{u}
$$

and then from the first equation in (A 5) we can conclude that

$$
\frac{\partial \tilde{n}}{\partial \tilde{x}}=-\frac{1}{\tilde{u}} \frac{\partial \tilde{\psi}}{\partial \tilde{s}}=-\frac{\partial \tilde{\psi} / \partial \tilde{s}}{\partial \tilde{\psi} / \partial \tilde{n}} .
$$


Equation (A 6) may be written as

$$
\tilde{u}(\tilde{x}, \tilde{\psi})=\frac{\partial \tilde{\psi}}{\partial \tilde{n}}[\tilde{s}(\tilde{x}, \tilde{\psi}), \tilde{n}(\tilde{x}, \tilde{\psi})] .
$$

Differentiating (A 8) with respect to $\tilde{x}$, we have

$$
\frac{\partial \tilde{u}}{\partial \tilde{x}}=\frac{\partial^{2} \tilde{\psi}}{\partial \tilde{s} \partial \tilde{n}}+\frac{\partial^{2} \tilde{\psi}}{\partial \tilde{n}^{2}} \frac{\partial \tilde{n}}{\partial \tilde{x}} .
$$

Using further (A 7), we can conclude that

$$
\frac{\partial \tilde{u}}{\partial \tilde{x}}=\frac{\partial^{2} \tilde{\psi}}{\partial \tilde{s} \partial \tilde{n}}-\frac{1}{\tilde{u}} \frac{\partial \tilde{\psi}}{\partial \tilde{s}} \frac{\partial^{2} \tilde{\psi}}{\partial \tilde{n}^{2}} .
$$

Differentiation of (A 8) with respect to $\tilde{\psi}$ results in

$$
\frac{\partial \tilde{u}}{\partial \tilde{\psi}}=\frac{\partial^{2} \tilde{\psi}}{\partial \tilde{n}^{2}} \frac{\partial \tilde{n}}{\partial \tilde{\psi}}=\frac{1}{\tilde{u}} \frac{\partial^{2} \tilde{\psi}}{\partial \tilde{n}^{2}},
$$

which means that

$$
\tilde{u} \frac{\partial \tilde{u}}{\partial \tilde{\psi}}=\frac{\partial^{2} \tilde{\psi}}{\partial \tilde{n}^{2}} .
$$

Similarly, if we differentiate (A 12) with respect to $\tilde{\psi}$, we see that

$$
\tilde{u} \frac{\partial}{\partial \tilde{\psi}}\left(\tilde{u} \frac{\partial \tilde{u}}{\partial \tilde{\psi}}\right)=\frac{\partial^{3} \tilde{\psi}}{\partial \tilde{n}^{3}} .
$$

Substitution of (A 10) and (A 13) into (4.125a) results in (4.129a). have

In order to deduce the initial condition (4.129b), we combine (4.126) with (A 6). We

$$
\frac{\partial \tilde{\psi}}{\partial \tilde{n}}=\mathrm{e}^{-\tilde{x} / 4} \sqrt{2+\frac{\tilde{\psi}^{2}}{\mathrm{e}^{-\tilde{x} / 2}}}
$$

and therefore,

$$
\tilde{n}=\int_{0}^{\tilde{\psi}} \frac{\mathrm{d} \tilde{\psi}^{\prime}}{\sqrt{2 \mathrm{e}^{-\tilde{x} / 2}+\tilde{\psi}^{\prime 2}}}=\sinh ^{-1}\left(\frac{\tilde{\psi}}{\sqrt{2} \mathrm{e}^{-\tilde{x} / 2}}\right) .
$$

The inversion of (A 15) results in (4.129b).

It remains to show that the interaction law $(4.129 d)$ holds. For this purpose we introduce function $\tilde{f}(\tilde{x})$ which gives the elevation of the 'dividing streamline' above the body surface. It may be determined by setting $\tilde{\psi}=-\infty$ in the second equation of (4.128), namely,

$$
\tilde{f}(\tilde{x})=-\left.\tilde{n}\right|_{\tilde{\psi}=-\infty}=\int_{-\infty}^{0} \frac{\mathrm{d} \tilde{\psi}}{\tilde{u}(\tilde{x}, \tilde{\psi})} .
$$

Integration of the continuity equation (4.125b) with the initial condition (4.127) yields

$$
\frac{\tilde{v}}{\tilde{u}}=\frac{\partial}{\partial \tilde{x}} \int_{-\infty}^{\tilde{\psi}} \frac{\mathrm{d} \tilde{\psi}^{\prime}}{\tilde{u}\left(\tilde{x}, \tilde{\psi}^{\prime}\right)}=\frac{\partial}{\partial \tilde{x}}\left[\tilde{f}(\tilde{x})+\int_{0}^{\tilde{\psi}} \frac{\mathrm{d} \tilde{\psi}^{\prime}}{\tilde{u}\left(\tilde{x}, \tilde{\psi}^{\prime}\right)}\right] .
$$

Applying the interaction law (4.125c) turns (A 17) into

$$
\frac{1}{2}=\frac{\mathrm{d} \tilde{f}}{\mathrm{~d} \tilde{x}}+\left.\frac{\partial \tilde{n}}{\partial \tilde{x}}\right|_{\tilde{\psi}=\infty}
$$


Since the solution for $\tilde{u}(\tilde{x}, \tilde{\psi})$ is symmetric with respect to the 'dividing streamline' $(\tilde{\psi}=0)$, we can claim that

$$
\left.\tilde{n}\right|_{\tilde{\psi}=\infty}=\tilde{f} .
$$

Consequently, (A 18) can be written as

$$
\frac{\partial \tilde{n}}{\partial \tilde{x}}=\frac{1}{4} .
$$

Combining (A 20) with (A 7) renders the interaction law in the form of (4.129d).

\section{REFERENCES}

ARAKI, D. 2006 Boundary-layer separation on a moving surface in supersonic flow. PhD thesis, The University of Manchester.

Battam, N. W., Gorounov, D. G., Korolev, G. L. \& Ruban, A. I. 2004 Shock wave interaction with a viscous wake in supersonic flow. J. Fluid Mech. 504, 301-341.

Blasius, H. 1908 Grenzschichten in flüssigkeiten mit kleiner reibung. Z. Math. Phys. 56 (1), 1-37 (translation in NACA TM 1256).

CEBECI, T. 1979 The laminar boundary layer on a circular cylinder started impulsively from rest. J. Comput Phys. 31, 153-172.

Cebeci, T. 1982 Unsteady separation. In Numerical and Physical Aspects of Aerodynamic Flows (ed. T. Cebeci), pp. 265-278. Springer.

CEBeCI, T. 1986 Unsteady boundary layers with an intelligent numerical scheme. J. Fluid Mech. 163, $129-140$.

Collins, W. M. \& Dennis, S. C. R. 1973a Flow past an impulsively started cylinder. J. Fluid Mech. 60, 105-127.

Collins, W. M. \& Dennis, S. C. R. $1973 b$ The initial flow past an impulsively started circular cylinder. Q. J. Mech. Appl. Maths 26, 53-75.

Cowley, S. J. 1983 Computer extension and analytic continuation of Blasius' expansion for impulsive flow past a circular cylinder. J. Fluid Mech. 135, 389-405.

Degani, A. T., Li, Q. \& WaLkeR, J. D. A. 1996 Unsteady separation from the leading edge of a thin airfoil. Phys. Fluids 8 (3), 704-714.

van Dommelen, L. L. \& Shen, S. F. 1980 The spontaneous generation of the singularity in a separating laminar boundary layer. J. Comput. Phys. 38 (2), 125-140.

van Dommelen, L. L. \& Shen, S. F. 1982 The genesis of separation. In Numerical and Physical Aspects of Aerodynamic Flows (ed. T. Cebeci), pp. 293-311. Springer.

van Dommelen, L. L. \& Shen, S. F. 1983 An unsteady interactive separation process. AIAA J. 21 (3), 358-362.

Elliott, J. W., Smith, F. T. \& Cowley, S. J. 1983 Breakdown of boundary layers: (i) on moving surfaces; (ii) in semi-similar unsteady flow; (iii) in fully unsteady flow. Geophys. Astrophys. Fluid Dyn. 25, 77-138.

Goldstein, S. 1948 On laminar boundary-layer flow near a position of separation. Q. J. Mech. Appl. Maths 1 (1), 43-69.

Goldstein, S. \& Rosenhead, L. N. 1936 Boundary layer growth. Proc. Camb. Phil. Soc. 32, 392-401.

Hartree, D. R. 1939 A solution of the laminar boundary-layer equation for retarded flow. Aero. Res. Coun. Rep. and Memo. 2426 (issued in 1949).

Howarth, L. 1938 On the solution of the laminar boundary layer equations. Proc. R. Soc. Lond. A 164, 547-579.

Kaplun, S. 1967 Mechanics and Singular Perturbations: A Collection of Papers. Academic.

Korolev, G. L., Gajuar, J. S. B. \& Ruban, A. I. 2002 Once again on the supersonic flow separation near a corner. J. Fluid Mech. 463, 173-199.

Koromilas, C. A. \& Telionis, D. P. 1980 Unsteady laminar separation: an experimental study. J. Fluid Mech. 97 (2), 347-384.

Landau, L. D. \& Lifshitz, E. M. 1944 Mechanics of Continuous Media. Gostekhizdat. 
Lighthill, M. J. 1953 On boundary layers and upstream influence. II. Supersonic flows without separation. Proc. R. Soc. Lond. A 217, 478-507.

Ludwig, G. R. 1964 An experimental investigation of laminar separation from a moving wall. AIAA Paper 64-6.

Messiter, A. F. 1970 Boundary-layer flow near the trailing edge of a flat plate. SIAM J. Appl. Math. 18 (1), 241-257.

Moore, F. K. 1958 On the separation of the unsteady laminar boundary layer. In Boundary Layer Research (ed. H. Görtler), pp. 296-311. Springer.

Nakayama, Y. 1988 Visualized Flow. Fluid Motion in Basic and Engineering Situations Revealed by Flow Visualization. Compiled by the Japan Society of Mechanical Engineers. Pergamon Press.

NeILAND, V. Y. 1969 Theory of laminar boundary layer separation in supersonic flow. Izv. Akad. Nauk SSSR, Mech. Zhidk. Gaza 53-57.

Peridier, V. J., Smith, F. T. \& Walker, J. D. A. 1991 Vortex-induced boundary-layer separation. Part 1. The unsteady limit problem $R e \rightarrow \infty$. J. Fluid Mech. 232, 99-131.

PrandTL, L. 1904 Über flüssigkeitsbewegung bei sehr kleiner Reibung. In Verh. III. Intern. Math. Kongr., Heidelberg, pp. 484-491. Teubner, 1905.

Proudman, I. \& Johnson, K. 1962 Boundary-layer growth near a rear stagnation point. J. Fluid Mech. 12, 161-168.

Puhak, R. I., Degani, A. T. \& Walker, J. D. A. 1995 Unsteady separation and heat transfer upstream of obstacles. J. Fluid Mech. 305, 1-27.

Robins, A. J. \& HowarTh, J. A. 1972 Boundary-layer development at a two-dimensional rear stagnation point. J. Fluid Mech. 56, 161-171.

RotT, N. 1956 Unsteady viscous flow in the vicinity of a stagnation point. Q. J. Appl. Math. 13, 444-451.

SeArs, W. R. 1956 Some recent developments in airfoil theory. J. Aeronaut. Sci. 23 (5), 490-499.

Stewartson, K. 1969 On the flow near the trailing edge of a flat plate. Mathematika 16 (1), 106-121.

Stewartson, K. \& Williams, P. G. 1969 Self-induced separation. Proc. R. Soc. Lond. A 312, 181-206.

Sychev, V. V., Ruban, A. I., Sychev, V. V. \& Korolev, G. L. 1998 Asymptotic Theory of Separated Flows. Cambridge University Press.

Sychev, V. V. 1972 Laminar separation. Mekh. Zhid. Gaza, No. 3, 47-59 (translation in Fluid Dyn. 7 (3), 407-417).

Sychev, V. V. 1978 Breakdown of plane laminar wake. Uch. Zap. TsAGI 9 (6), 9-16.

SycheV, V. V. 1979 Asymptotic theory of nonstationary separation. Mekh. Zhid. Gaza, No. 6, 21-32 (translation in Fluid Dyn. 14 (6), 829-838).

SycheV, V. V. 1980 On certain singularities in solutions of the boundary-layer equations on a moving surface. Prikl. Mat. Mekh. 44 (5), 831-838 (translation in J. Appl. Math. Mech. 44 (5), 587-591).

Sychev, V. V. 1984 On the asymptotic theory of laminar separation from a moving surface. Prikl. Mat. Mekh. 48 (2), 247-253 (translation in J. Appl. Math. Mech. 48 (2), 171-176).

SycheV, V. V. 1987 Analytical solution of the problem of flow near the boundary-layer separation point on a moving wall. Prikl. Mat. Mekh. 51 (3), 519-521 (translation in J. Appl. Math. Mech. 51 (3), 405-407).

Telionis, D. P. \& Tsahalis, D. T. 1974 Unsteady laminar separation over impulsively moved cylinders. Acta Astronautica 1, 1487-1505.

Telionis, D. P. \& Werle, M. J. 1973 Boundary layer separation from moving boundaries. J. Appl. Mech. 95, 369-374.

WANG, K. C. 1979 Unsteady boundary layer separation. Tech. Rep. MML TR 79-16C. Martin Marietta Laboratory, Baltimore, Maryland, USA.

WANG, K. C. 1982 On the current controversy about unsteady separation. In Numerical and Physical Aspects of Aerodynamic Flows (ed. T. Cebeci), pp. 279-291. Springer.

WUNDT, H. 1955 Wachstum der laminaren Grenzchicht an schräg angeströmten Zylinder bei Anfahrt aus der Ruhe. Ing.-Arch 23, 212-230. 